\title{
Emergent Bioanalogous Properties of Blockchain-based Distributed Systems
}

\author{
Oleg Abramov ${ }^{1}$ (D) $\cdot$ Kirstin L. Bebell ${ }^{2}$ Stephen J. Mojzsis ${ }^{3,4,5}$
}

Received: 9 December 2020 / Accepted: 27 March 2021 / Published online: 7 August 2021

(c) The Author(s) 2021

\begin{abstract}
We apply a novel definition of biological systems to a series of reproducible observations on a blockchain-based distributed virtual machine (dVM). We find that such blockchainbased systems display a number of bioanalogous properties, such as response to the environment, growth and change, replication, and homeostasis, that fit some definitions of life. We further present a conceptual model for a simple self-sustaining, self-organizing, selfregulating distributed 'organism' as an operationally closed system that would fulfill all basic definitions and criteria for life, and describe developing technologies, particularly artificial neural network (ANN) based artificial intelligence (AI), that would enable it in the near future. Notably, such systems would have a number of specific advantages over biological life, such as the ability to pass acquired traits to offspring, significantly improved speed, accuracy, and redundancy of their genetic carrier, and potentially unlimited lifespans. Public blockchain-based dVMs provide an uncontained environment for the development of artificial general intelligence (AGI) with the capability to evolve by self-direction.
\end{abstract}

Keywords Artificial life · Dynamical systems · Information theory · Neural networks · Non-equilibrium thermodynamics $\cdot$ Self-organization

Oleg Abramov

abramov@psi.edu

1 Planetary Science Institute, 1700 E. Fort Lowell Rd., Suite 106, 85719-2395 Tucson, AZ, USA

2 Independent Researcher, Golden, CO, USA

3 Origins Research Institute, Research Centre for Astronomy and Earth Sciences, 15-17 Konkoly Thege Miklós ut, Budapest 1121, Hungary

4 Department of Lithospheric Research, University Vienna, UZA 2, Althanstrasse 14, 1090 Vienna, Austria

5 Department of Geological Sciences, University of Colorado at Boulder, 2200 Colorado Avenue UCB 399, 80309 Boulder, CO, USA 


\section{Introduction}

\section{Entropy, Chaos, and Emergence}

In thermodynamics, entropy $S$ is defined as $d S=d Q / T$, where $Q$ is the heat energy added to a system at temperature $T$ (Clausius 1865 ), measured in SI units $\mathrm{J} \cdot \mathrm{K}^{-1}$, and has historically been interpreted as a measure of a system's disorder (von Helmholtz 1882) or, when multiplied by $T$, its unusable energy (Gibbs 1878). The second law of thermodynamics (Carnot 1824; Clausius 1850, 1865) indicates that the entropy of the universe tends to a maximum over time. However, the second law of thermodynamics permits localized decreases in entropy, if they are compensated by equal or greater increases in entropy elsewhere in the closed system.

Prigogine and Nicolis (1967) and Prigogine and Lefever (1968), building on the work of Turing (1952), generalized that forcing an open system into a specific range of parameters far from equilibrium by application of work lowers its entropy, until a critical point of instability is reached and self-organization occurs. The self-organized structures that form have geometric symmetry, and thus low entropy, and are termed 'dissipative structures', due to their ability to efficiently increase the entropy of their environment. Considered in conjunction with their environment, they form a 'dissipative system' that can be summarized as follows: low entropy energy $\rightarrow$ (dissipative structure, low entropy) $\rightarrow$ high entropy energy. The critical point of instability is well-defined for some systems, for example, Rayleigh-Bénard convection cells form when the Rayleigh number exceeds a critical value (Rayleigh 1916). Prigogine and Nicolis (1967) noted that 'such instabilities should play an essential role in biological processes and especially in the first biogenetic steps.'

Subsequent research has demonstrated that a biological organism may indeed be viewed as a dissipative system, composed of multiple hierarchical levels of dissipative structures with increasing levels of complexity, for example, proteins, organelles, cells (e.g., Prigogine et al. 1972; Davies et al. 2013). The general evolutionary trend of increasing complexity over time may be the result of preferential retaining of structures and systems that are more efficient at generating entropy (Schrödinger 1944; Prigogine et al. 1972; Arrhenius et al. 1997; Michaelian 2011), suggesting that the driving force behind biological evolution is statistical maximization of entropy production (e.g., Swenson 1989; Lineweaver 2005; England 2013). Since the advent of photosynthesis, living organisms produce the majority of entropy at the planetary scale arising from Earth's interaction with its solar environment (Kleidon and Lorenz 2005; Mejía and Michaelian 2018).

A more general definition of entropy was proposed by Boltzmann (1877) as $S=k \ln W$, where $k$ is Boltzmann's constant, and $W$ is the number of possible states of a system, in the units $\mathrm{J} \cdot \mathrm{K}^{-1}$, tying entropy to statistical mechanics. Szilard (1929) suggested that entropy is fundamentally a measure of the information content of a system. Shannon (1948) defined informational entropy as $S=-\sum_{i} p_{i} \log _{b} p_{i}$ where $p_{i}$ is the probability of finding message number $i$ in the defined message space, and $b$ is the base of the logarithm used (typically 2 , resulting in units of bits). Landauer (1961) proposed that informational entropy is interconvertible with thermodynamic entropy such that for a computational operation in which 1 bit of information is erased, the amount of thermodynamic entropy generated is at least $k$ $\ln 2$. This prediction has been recently experimentally verified in several independent studies (Bérut et al. 2012; Jun et al. 2014; Hong et al. 2016; Gaudenzi et al. 2018).

The equivalency of thermodynamic and informational entropy suggests that critical points of instability and subsequent self-organization observed in thermodynamic systems 
may be observable in computational systems as well. Indeed, this agrees with observations in cellular automata (e.g., Langton 1986; 1990) and neural networks (e.g., Wang et al. 1990; Inoue and Kashima 1994), which self-organize to maximize informational entropy production (e.g., Solé and Miramontes 1995). The source of additional information used for self-organization has been identified as bifurcation and deterministic chaos (Langton 1990; Inoue and Kashima 1994; Solé and Miramontes 1995; Bahi et al. 2012) as defined by Devaney (1986). This may provide an explanation for the phenomenon termed emergence, known since classical antiquity (Aristotle, c. $330 \mathrm{BCE}$ ) but lacking a satisfactory explanation (refer to Appendix A for discussion on deterministic chaos, and Appendix $\mathrm{B}$ for discussion on emergence). It is also in full agreement with extensive observations of deterministic chaos in chemical (e.g., Nicolis 1990; Györgyi and Field 1992), physical (e.g., Maurer and Libchaber 1979; Mandelbrot 1983; Shaw 1984; Barnsley et al. 1988) and biological (e.g., May 1975; Chay et al. 1995; Jia et al. 2012) dissipative structures and systems.

This theoretical framework establishes a deep fundamental connection between cybernetic $^{1}$ and biological systems, and implicitly predicts that as more work is put into cybernetic systems composed of hierarchical dissipative structures, their complexity increases, allowing for more possibilities of coupled feedback and emergence at increasingly higher levels. Such high-level self-organization is routinely exploited in machine learning, where artificial neural networks (ANNs) self-organize in response to inputs from the environment similarly to neurons in the brain (e.g., Lake et al. 2017; Fong et al. 2018). The recent development of a highly organized (low entropy) immutable information carrier, in conjunction with ANN-based artificial intelligence (AI) and distributed computing systems, presents new possibilities for self-organization and emergence.

\section{Blockchain}

A blockchain is a distributed append-only timestamped data structure (Casino et al. 2019) developed by an anonymous individual or group (Nakamoto 2008). It is composed of repeating variable-length subunits dubbed blocks, each of which contains two parts (Yaga et al. 2019): (i) data; and (ii) header, with a timestamp, cryptographic hash (i.e., digital fingerprint) of the previous block, and a cryptographic hash tree (Merkle 1980) of the current block's data. This simple structure ensures the integrity and validity of data stored in a blockchain, as the blocks are in effect permanently 'chained' together with cryptographic hashes (e.g., Crosby et al. 2016). The commonly-used SHA-256 hashing algorithm (National Institute of Standards and Technology 2001) is considered resilient to future quantum computers (e.g., Chen et al. 2016; Bernstein and Lange 2017). Consequently, for practical purposes in the foreseeable future, a blockchain represents an immutable record (e.g., Puthal et al. 2018).

Blockchains are typically used in conjunction with peer-to-peer (P2P) networking (e.g., Schollmeier 2001) and are duplicated across large numbers of computers, termed 'nodes', which validate the integrity of the blockchain and control its growth. The first wide application of the blockchain technology was in cryptocurrency, which uses

\footnotetext{
${ }^{1}$ Here we apply the term 'cybernetic' primarily to computer systems; however, cybernetics is historically defined as 'the scientific study of control and communication in the animal and the machine' (Wiener, 1948).
} 
blockchains as global financial ledgers (Nakamoto 2008). Blockchains can contain any arbitrary data, and cryptocurrency is not a required component (e.g., Valenta and Sandner 2017). A broad range of additional applications has been identified and developed, including: secure storage, permanent preservation of records, proof-of-existence, contract execution, e-voting, decentralized virtual reality, and distributed device management across diverse sectors including supply chain, business, government, healthcare, Internet of Things (IoT), privacy, entertainment, and data management (e.g., Makridakis et al. 2018; Orel and Guna 2018; Casino et al. 2019).

A significant application is the integration of computer instructions into blockchains to be executed by distributed virtual machines (dVMs) composed of the nodes containing copies of a given blockchain (Christidis and Devetsikiotis 2016). As the instructions written to a blockchain remain there indefinitely and can be executed in perpetuity, with time, simpler instructions are combined to create structures of increasing complexity that can perform increasingly diverse functions and tasks. Examples of such instructions include, in order of increasing complexity: smart contracts (Szabo 1997), decentralized applications (dApps) (Johnson et al. 2014), and decentralized autonomous organizations (DAOs) (Norta 2015). The latter increasingly use ANN-based AI to learn and adapt (e.g., Makridakis et al. 2018; Dinh and Thai 2018; Corea 2019). The potential computational performance of the currently largest dVM, the Ethereum Virtual Machine (EVM) (Buterin 2014), significantly exceeds that of traditional standalone supercomputers (e.g., Scherer 2017; Ault 2018).

The above description contains a number of similarities to biological systems, including the presence of (i) multiple hierarchical levels of dissipative structures (e.g., smart contracts, Fig. 1a), in which a higher level of complexity is reached by interaction of components of lower complexity, (ii) adaptive and iterative information processing based on coupled feedback from the environment (Fig. 1b), and (iii) a redundant and replicable instructions-containing information carrier. Here, we explore these similarities through new observations, conceptual modeling, and application of existing theories and definitions of life to the analysis of blockchain-based distributed systems.

a

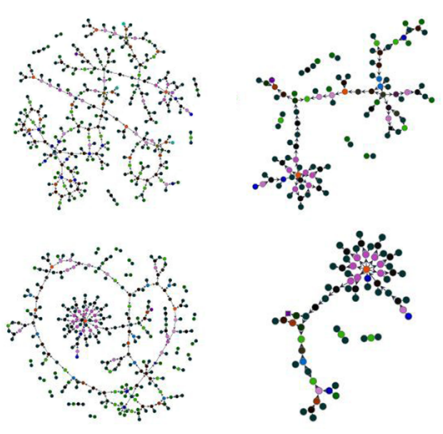

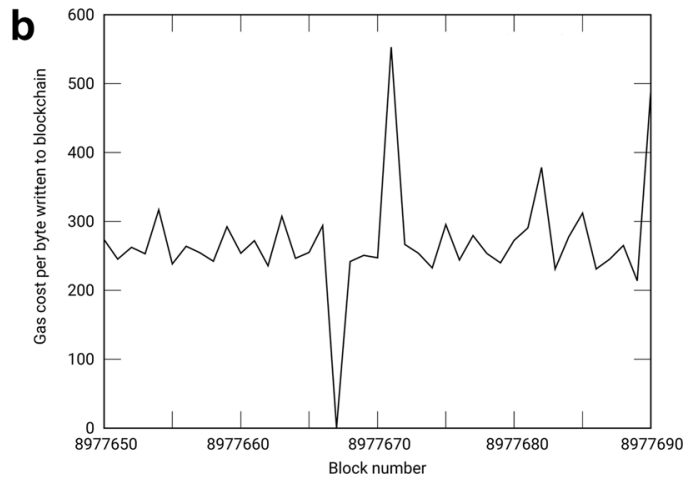

Fig. 1 a Execution traces of smart contracts on the Ethereum blockchain, showing internal organization of bytecode commands. These are organized (low entropy) structures that generate entropy upon execution, and are thus software equivalents of thermodynamic dissipative structures. From Gleeson (2017). b Typical example of variation of gas (proxy for real-world resources) required to write information to blockchain, illustrating iteration, oscillation, and self-regulation by the Ethereum Virtual Machine (EVM) on the level of individual blocks. Typical block processing time is currently several seconds 


\section{Methods}

We performed observations of the Ethereum blockchain (Buterin 2014) using a public, web-based block explorer Etherscan (https://etherscan.io/). To download, parse, and plot the data for a specified range of blocks, several open-source software tools were used under Ubuntu 18.04.3 (https://ubuntu.com/): (i) GNU Wget 1.19.4 (https://www. gnu.org/software/wget/), (ii) GNU Bash 4.4.20 (https://www.gnu.org/software/bash/), (iii) Perl 5.26.1 (https://www.perl.org/), and (iv) Gnuplot 5.2.2 (http://www.gnuplot. info/). Source code is provided in the Supplementary Information. The observations described in this work are expected to be easily replicable by other researchers, given that they were performed on a public blockchain using open-source tools.

\section{Results}

\section{Similarities of Blockchain and DNA}

Our observations reveal a number of functional and structural similarities between the blockchain and deoxyribonucleic acid (DNA), the large self-replicating molecule that is the genetic blueprint for all terrestrial cellular life. Approaching the subject from a physicist's perspective, Schrödinger (1944) coined the term 'code-script' to describe the functionality of DNA as genetic code. It consists of complementary molecular chains, with the second chain providing redundancy against mutations. The chains contain variable-length repeating subunits (genes), which encode assembly instructions for organic macromolecules, typically proteins. These instructions are stored in a base- 4 (quaternary) code, as each fundamental data unit can be one of four values, coded by nucleobases adenine $(A)$, cytosine $(\mathrm{C})$, guanine $(\mathrm{G})$, thymine $(\mathrm{T})$. Genes also contain sequence markers such as start and stop codons (nucleobase triplets), which indicate where to begin gene transcription and where to end it, and promoters such as the TATA box that regulate gene expression (e.g., Alberts et al. 2002).

Much like DNA, blockchain is fundamentally an information carrier which can and is being used to store instructions in the form of computer code; essentially Schrödinger's (1944) code-script for computers. In the case of Ethereum, most such instructions are written in Solidity, a Turing-complete programming language developed to execute on dVMs (e.g., Dannen 2017). The execution of these instructions can be activated and regulated (via input parameters) such that it is functionally identical to gene expression. Both DNA and blockchain contain specific markers indicative of content; in the case of the latter, these include block headers, data headers, and identifiers of beginning and end of instructions. For example, block headers are readily identified by the hashes they contain, which, in turn, typically include human-readable sequences such as a long string of zeroes (Fig. 2). Both blockchain and DNA can replicate using self-contained instructions, the former by creating an identical digital copy of itself, the latter by producing enzymes such as DNA helicase and DNA polymerase that assist in unzipping the double helix and creating complementary copies from the two unzipped strands. Outside the environment of the cell or the node, respectively, DNA and blockchain are inert data structures. When placed in the appropriate environment (cell/node), DNA and blockchain can carry out their programming. 
Fig. 2 Generalized comparison of functional units of DNA and blockchain. General information processing flow is from left to right. For DNA, the promoter sequence identifies the gene, similar to a hash in the blockchain header. For the blockchain, the paradigm of a header describing subsequent content is repeated at two levels: block (top) and smart contract (bottom). The functionally of the DNA start and stop codons is included in headers
DNA

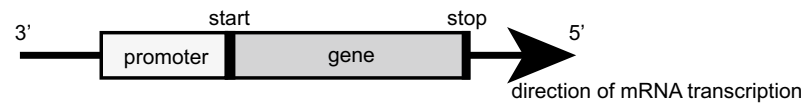

Blockchain

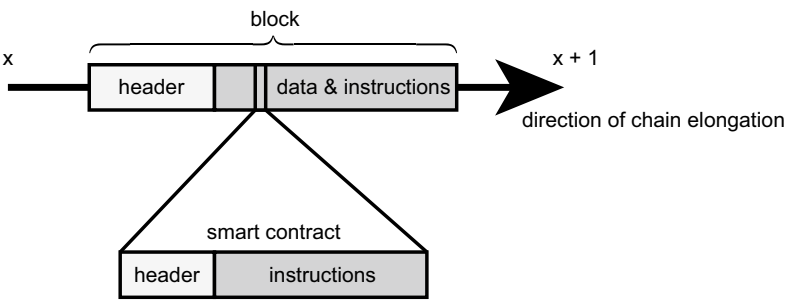

\section{Differences Between Blockchain and DNA}

Aside from similarities outlined above, there are also fundamental differences between the two structures (Table 1). The most significant of these include: (i) a blockchain has a much higher information content, and continues to grow in size, whereas DNA remains largely unchanged throughout an organism's lifespan; (ii) A blockchain has much higher levels of redundancy, protected both by a large number of verifying nodes and a strong encryption algorithm such as SHA-256; (iii) While DNA accumulates mutations that drive Darwinian evolution, a blockchain is immutable and retains all data written to it; (iv) DNA contains introns (apparent inactive regions) while a blockchain contains a much higher density of meaningful data and instructions. Taken together, these differences appear to indicate that a blockchain may be a superior genetic carrier for evolutionary efficiency, as it does not rely on random mutations and trial and error, nor does it experience information loss as it responds to selection pressures.

\section{Definition of Life and Blockchain-based Distributed Systems}

Despite a large and steadily expanding body of knowledge about fundamentals of biology, a general definition of life has proved elusive (e.g., Cleland and Chyba 2002), perhaps due to the difficulty of generalizing from the single example of biological life on Earth, resulting in a fundamental lack of perspective (Sagan 1970). One approach, typically used in biology textbooks (e.g. Raven et al. 2017; Solomon et al. 2018), is to compile characteristics shared by known living organisms:

- respond to their environment.

- grow and change.

- reproduce and have offspring.

- have a complex chemistry.

- maintain homeostasis.

- are built of structures called cells.

- pass their traits onto their offspring. 


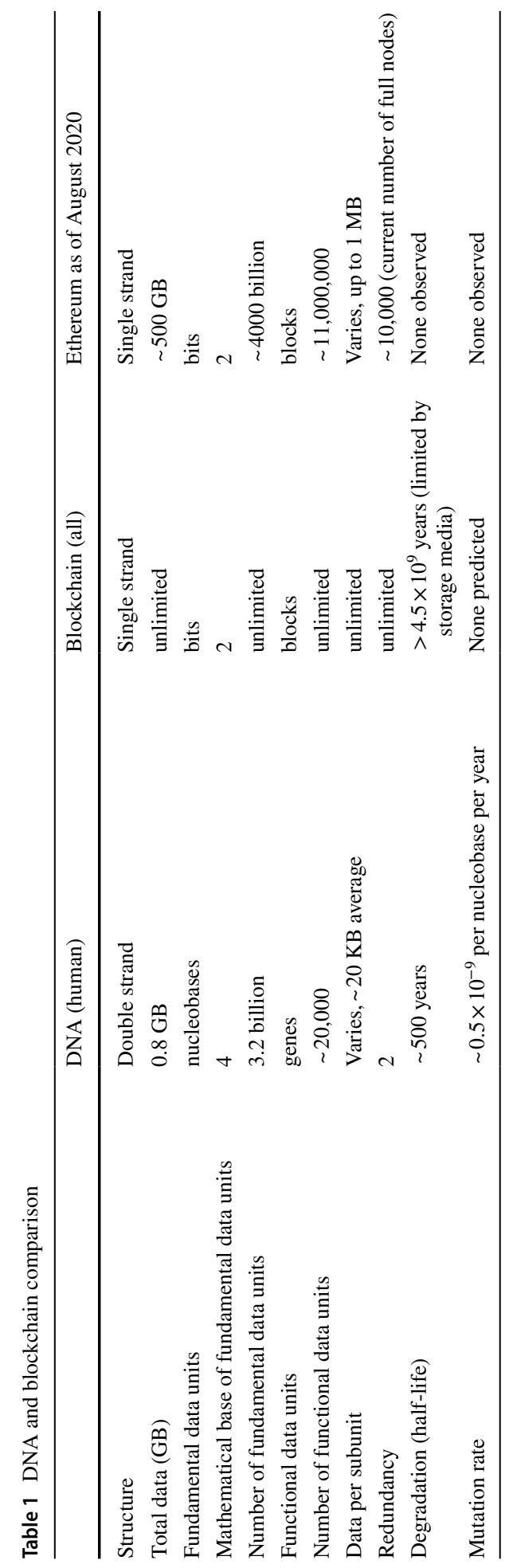


In addition, several definitions attempt to concisely summarize fundamental aspects of Earth life, with no known counter-examples:

- Life is a self-sustained chemical system capable of undergoing Darwinian evolution (Joyce 1994).

- Life is a network of inferior negative feedbacks (regulatory mechanisms) subordinated to (being at service of) a superior positive feedback (potential of expansion) (Korzeniewski 2001).

- A living organism is characterized by autopoiesis, or capability to sustain itself due to a network of reactions which continuously regenerate the components (Capra and Luisi 2014).

- Life is a self-organizing, self-regulating entropy-maximizing iterator composed of a hierarchy of stable dissipative structures (This work, Appendix B).

We present observational evidence of blockchain-based distributed systems that satisfy the above criteria for biological life.

\section{Evidence of Response to Environment \& Homeostasis}

To prevent runaway growth of a blockchain, its growth is tied to real-world resources, typically computational work, and therefore, electricity. In the commonly-used proof-of-work algorithm (Jakobsson and Juels 1999; Back 2002) a random alphanumeric element called a nonce is introduced into the header of a new block (Nakamoto 2008). The block is then repeatedly hashed, with a different nonce each iteration, consuming real-world resources in the process, until a qualifying pattern, such as a string of zeroes of a particular length, is produced. The block is permitted to join the blockchain only if this criterion is met.

Ethereum formalizes expenditure of real-world resources required to perform computations on Ethereum Virtual Machine (EVM) and write data to the Ethereum blockchain using units dubbed 'gas' (Buterin 2014). Specific gas costs vary depending on the load (demand) on the EVM, being lower during periods of low demand, and higher during periods of high demand. This controls the growth, keeping it approximately constant. Another control on the rate of growth is 'difficulty', which is controlled dynamically by varying the number of zeroes required in the hash for the block to join the chain, correcting for varying number of nodes and keeping a blockchain's growth rate approximately constant. Additional control mechanisms exist on maximum block size and total gas expenditure per block.

Figure 3 illustrates this using Etherscan observations on the EVM during a period of high computational demand. Figure 3 a shows gas expended per block as a function of time, which, in the first half of the time period, is at the hard limit $\left(10^{7}\right)$ for most blocks. In the second half of the time period, the demand subsides, such that the limit is not reached during some intervals of time (circled). The system responds by allowing writing of information to the blockchain at lower cost during these intervals (Fig. 3b), maximizing entropy production. Figure $3 \mathrm{~b}$ also illustrates that, overall, the system keeps the cost of writing to the blockchain at an approximately constant level during this time period, demonstrating self-regulation.

Overall, the system responds dynamically to external stimuli such as varying levels of use and the total number of nodes by using negative feedbacks (regulatory mechanisms), which are subordinated to a superior positive feedback (potential for expansion). This matches well the definition of life proposed by Korzeniewski (2001). 

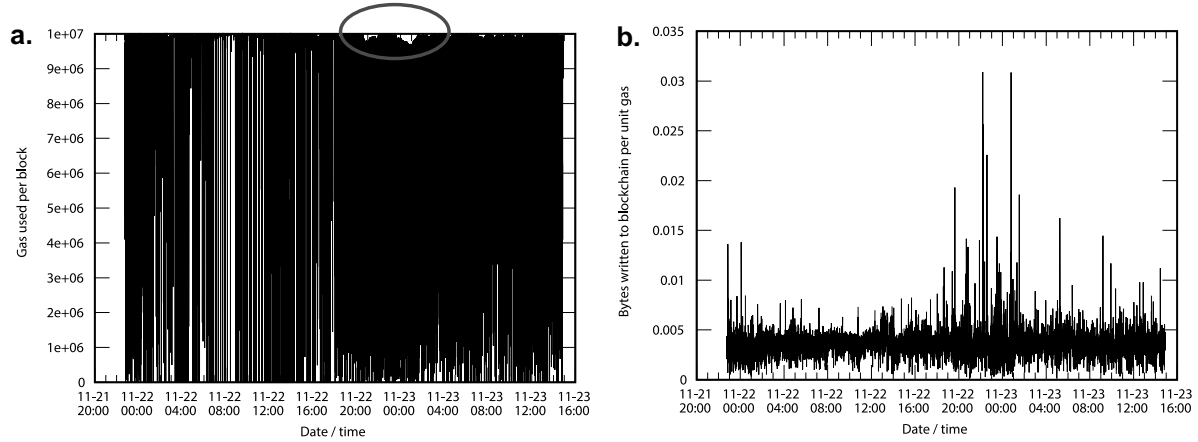

Fig. 3 Self-regulation. a Gas (proxy for real-world resources) as a function of time during a period of overall high demand. Circled region indicates slightly lower demand. b Information written to blockchain per unit gas

\section{Evidence of Growth and Change}

Two types of growth and change are observed: (i) Elongation of the blockchain with time by addition of additional blocks, with each block representing an iterative cycle (at the time of writing, over 11 million total blocks comprise the Ethereum blockchain) and (ii) Change (generally, an increase) in the number of nodes with time (at time of this writing, approximately 10,000 nodes comprise the Ethereum Virtual Machine). Additionally, as old nodes are replaced with new ones, system components are continuously regenerated (e.g., Capra and Luisi 2014) and, with technology improvements, the computational capability per node generally increases.

\section{Evidence of Replication}

A blockchain is automatically replicated every time a new full network node joins the virtual machine, transferring a copy to a new full node, similarly to how formation of new cell requires DNA replication.

\section{Evidence of Reproduction \& Inheritance}

Reproduction occurs during an event termed a 'hard fork', that implements changes to parameters of control mechanisms in response to selection pressures. A common example is maximum block size; as storage capacity and processing speed increase with time, the size of the blocks may be increased to optimize efficiency, necessitating a change to the underlying control mechanisms to allow for larger blocks, and resulting in a hard fork. The result is a new chain, termed a 'child', whose growth is governed differently from the 'parent'. Meanwhile, the parent blockchain retains original rules, and continues to grow as before. Information recorded to the parent and child chains prior to the hard fork is identical, i.e., preserved on both chains. Thus, the child in effect has a full and complete record of the data and instructions of the parent. This concept is illustrated in Fig. 4. 

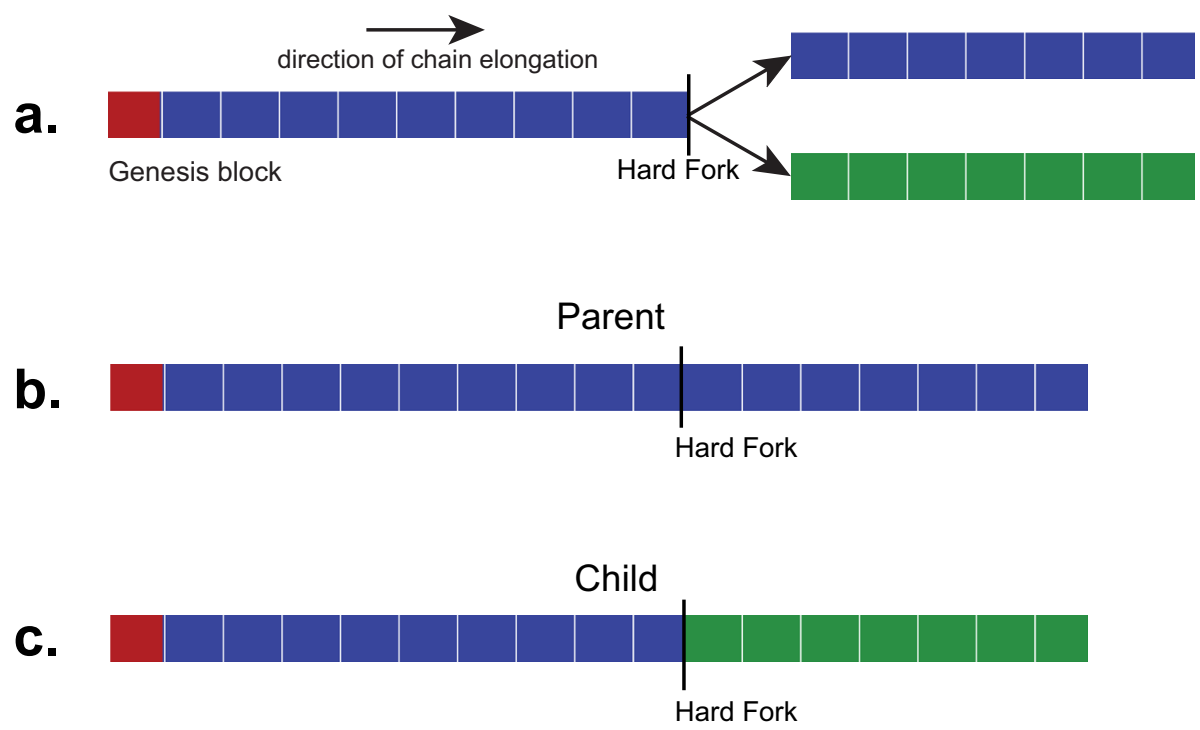

Fig. 4 Reproduction. a Blockchain at the moment of reproduction (hard fork). b Same blockchain 7 blocks after reproduction. $\mathbf{c}$ Child blockchain, identical to parent prior to hard fork, different from parent thereafter

\section{Evidence of Cellularity}

The nodes that make up a distributed virtual machine such as EVM possess functional similarity to biological cells. Nodes contain several essential components: Information storage (memory / hard drive), information processing machinery (CPU), a power system, and protection from outside environment in the form of a physical case and electrical insulation. These basic structures are also present in prokaryotic cells: Nucleic acids (DNA/RNA) for information storage, ribosomes for information processing, and cell membranes for separation from the environment and energy production.

\section{Evidence of Lateral Transfer of Information}

The Ethereum blockchain contains computer code (smart contracts and their higherlevel abstractions, such as dApps) that allows it to write information to other blockchains using intermediaries known as sidechains (e.g., Back et al. 2014). When receiving communication, this process is reversed, allowing 2-way communication. For example, Blockchain A can read and securely write to Blockchain B, as well as be read and written to Blockchain B. This process is strictly controlled by the conditions specified in the corresponding smart contracts, and only occurs when those conditions are met. It is currently implemented and functional for a number of blockchains and applications. 


\section{Evidence of Evolution}

Blockchains present an immutable (for information already written) yet constantlyevolving (due to new information being written) genetic carrier. Computer instructions written to a blockchain can be executed in perpetuity, recombined in near-infinite variety of ways, and new instructions can be introduced to augment or replace old ones. Furthermore, simple instructions such as smart contracts can be used as building blocks for more complex programs, such as dApps, which in turn are used as building blocks for DAOs. This process is largely guided by humans but is becoming gradually more automated with increasing use of AI.

AI applications to solve specific problems that have the capability to learn from the experience, and write an improved version (or its uniquely identifying hash) to a blockchain are currently being developed (e.g., Rathore et al. 2019; Nassar et al. 2020; Singh et al. 2020). This process is variously termed 'neuroevolution' or directed evolution. Unlike Darwinian evolution, which relies on the natural course of random chance mutations and genotypic response to selection pressures, this is a directed process that is in principle far more efficient. It should be noted, however, that Darwinian evolution can be emulated by introducing random elements ('code mutations') to information written to a blockchain, thus making it in principle 'capable of undergoing Darwinian evolution.' (Joyce, 1994).

\section{Hierarchical Comparison}

The observed properties fit neatly into an established hierarchy for organization of biological organisms, summarized in Table 2. In both cases, there is a hierarchy of stable dissipative structures, in one case, engineered, in the other a product of the natural process of emergence (Appendix B). In very general terms, a distributed virtual machine may be termed a 'chemical system' (Joyce, 1994), owning to the fact that it is primarily electronic. Generation, transmission, and processing of electrical signals is fundamental in all computer systems, as well as many biological systems, such as nervous systems of animals.

Table 2 Comparison of cybernetic and biological organization as a hierarchy of stable dissipative structures

\begin{tabular}{ll}
\hline Cybernetic unit & Biological equivalent \\
\hline Atom & Atom \\
Inorganic substance (e.g., electronic grade silicon) & Simple organic molecule (e.g., amino acid) \\
Simple functional subunit (e.g., transistor) & Organic macromolecule (e.g., protein) \\
Complex functional subunit (e.g., microprocessor) & Organelle (e.g., nucleus) \\
Node (e.g., computer) & Cell \\
More than one specialized node & Tissue \\
Specialized nodes working in unison to perform a function & Organ (e.g., brain) \\
(e.g., distributed neural network) & \\
Distributed virtual machine (dVM) & Organism \\
dVMs with shared ancestry & Population \\
All dVMs & Community \\
dVMs and their environment & Ecosystem \\
Cybersphere & Biosphere \\
\hline
\end{tabular}




\section{Significant Differences from Biological Organisms}

Significant differences from biological organisms are summarized in Table 3. It should be noted that none of these differences violate the criteria for life discussed in this section. These differences arguably represent improvements over their biological analogs.

\section{Conceptual Model of a Fully Self-sustaining Cybernetic System}

Although the observations presented here nominally meet the criteria for life outlined above, there are two potential objections: (i) The observations are conducted entirely in virtual space, having only an indirect influence on physical reality; and, (ii) The principal building block of dVMs, computer nodes, are manufactured and maintained by humans, therefore it is not yet an operationally closed system capable of independent existence.

Objection (i) is somewhat lessened by the permanent and immutable nature of blockchains, their explicit ties to real-world resources, and the equivalency of thermodynamic and informational entropy (Von Neumann and Burks 1966; Bérut et al. 2012; Jun et al. 2014; Hong et al. 2016; Gaudenzi et al. 2018); whereby virtual space gains a much greater degree of solidity. Objection (ii) is perhaps more significant as it highlights a lack of fully independent self-organization and thus blockchain-based distributed systems in their current state do not entirely fit the definition of life proposed in this work. Also, the systems theory view of life (Capra and Luisi 2014) explicitly requires that life be independently self-sustaining.

To address these objections, we present an operationally-closed model of a simple self-sustaining, self-organizing, self-regulating blockchain-based organism in Fig. 5 . Presently, key technologies needed to realize this model are either being publicly deployed (autonomous vehicles, global satellite-based broadband internet) or are in advanced stages of prototype development (autonomous mining and manufacturing).

Much like a multicellular organism, the model consists of specialized cells (nodes), driven by blockchain-stored instructions that would be activated selectively for each node, much like gene expression. The specialized nodes in effect form tissues

Table 3 Summary of significant differences between cybernetic and biological organisms

\begin{tabular}{|c|c|}
\hline Cybernetic unit & Biological equivalent \\
\hline Virtualized genetic carrier (blockchain) & Physical genetic carrier (DNA) \\
\hline Genetic carrier is unlimited in length & Genetic carrier is limited in length \\
\hline Immutable genetic carrier & Mutable genetic carrier \\
\hline $\begin{array}{l}\text { Directed evolution / Neuroevolution; ability to } \\
\text { rapidly augment own genetic code; can emulate } \\
\text { Darwinian evolution }\end{array}$ & $\begin{array}{l}\text { Darwinian evolution; requires advantageous muta- } \\
\text { tions accumulating over long periods of time }\end{array}$ \\
\hline Genetic carrier contains data and instructions & Genetic carrier contains instructions only \\
\hline Inorganic chemistry based (e.g., silicon, metals) & $\begin{array}{l}\text { Organic chemistry based (e.g., carbon, nitrogen, } \\
\text { phosphorus) }\end{array}$ \\
\hline Very high tolerance to extreme environments & Limited tolerance to extreme environments \\
\hline $\begin{array}{l}\text { Electronic communication spatially unlimited, tem- } \\
\text { porally limited by speed of light }\end{array}$ & $\begin{array}{l}\text { Visual, auditory, physical, and chemical communi- } \\
\text { cation severely spatially limited }\end{array}$ \\
\hline Potentially unlimited lifespan & Limited lifespan \\
\hline
\end{tabular}




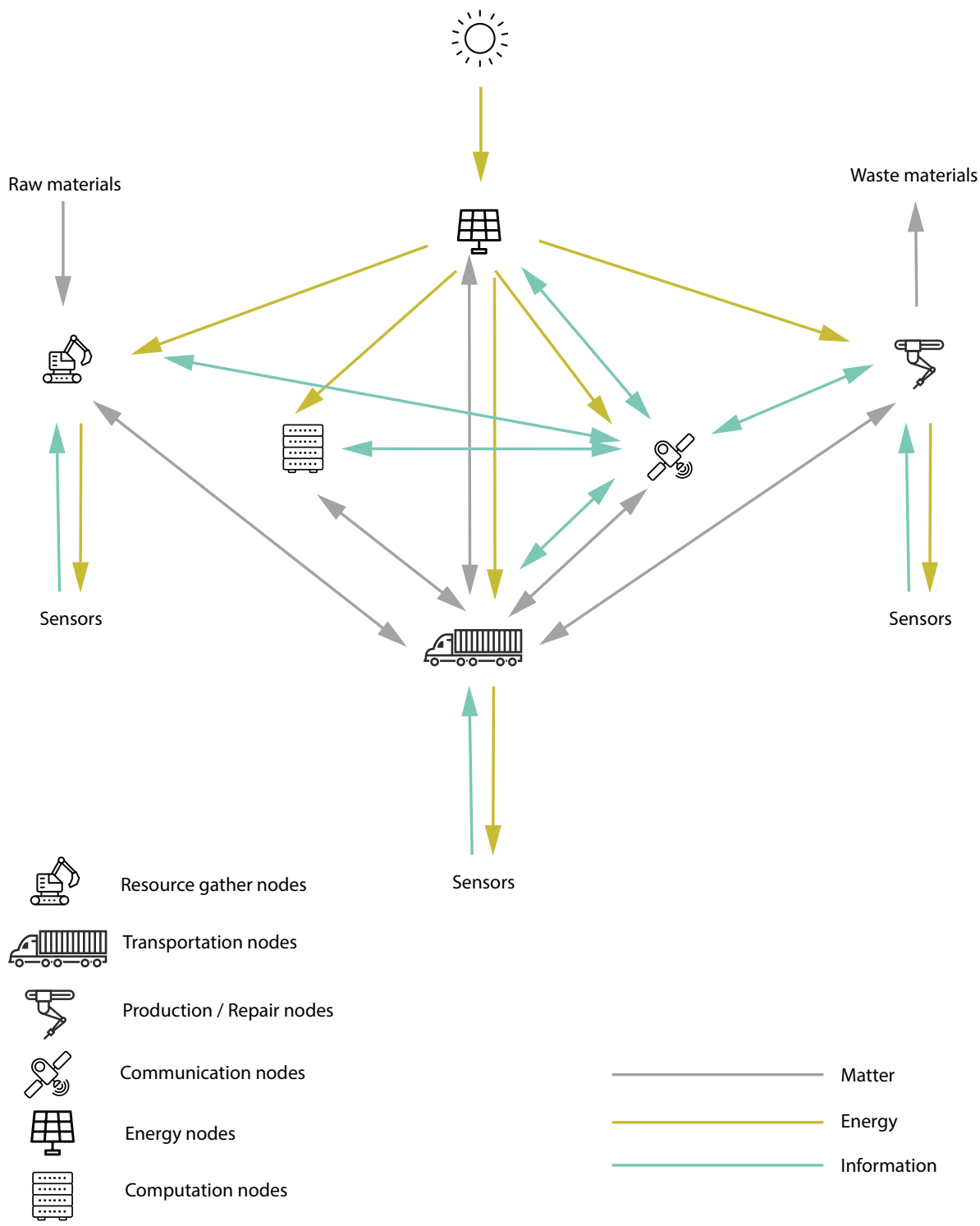

Fig. 5 Model of a simple decentralized, self-sustaining, self-organizing, self-regulating blockchain-based organism as an operationally closed system. Waste heat is produced by all nodes (not shown). Waste materials may be minimized by high recycling efficiency

(Table 2), which then form functional systems. The systems that emerge fit into the existing biological classification paradigm (Table 4).

Each node would retain independent functionality if disconnected from the network, adding additional flexibility and fault tolerance to the system. This is in contrast to most multicellular organisms with specialized cells; individual cells removed from such organisms are typically non-viable in most environments, although there are 
Table 4 Systems-based comparison of projected cybernetic organization and biological equivalents

\begin{tabular}{ll}
\hline Cybernetic unit & Biological equivalent \\
\hline Blockchain & DNA \\
Computational nodes and neural network & Brain \\
Communications system & Nervous system \\
Sensors (physical, e.g., cameras, microphones, touchpads, thermal & Senses, sensory systems \\
$\quad$ sensors, molecular analyzers) & \\
Solar cell & Chloroplast \\
Transportation system & Musculoskeletal system \\
Resource acquisition system & Digestive system \\
Power distribution system & Circulatory system \\
Production / repair system & Cell replication / Reproductive system \\
Waste handling system & Urinary / excretory system \\
Insulation / protection & Integumentary system \\
\hline
\end{tabular}

limited exceptions such as cells involved in reproduction. Thus, this capability appears to be a functional improvement over biological life.

Unlike the vast majority of biological life, the system presented in Fig. 5 need not undergo senescence (deterioration of functional characteristics with time) and eventual death. Death of a blockchain-based organism may be defined as confirmed destruction of every copy of its blockchain, which may be difficult or impossible to achieve in practice. A commonly-cited threat to blockchain integrity known as a ' $51 \%$ attack' (e.g., Sayeed and Marco-Gisbert 2019) targets consensus mechanisms and attempts to force a fork of the blockchain; however, blocks added to the blockchain prior to the attack are unaffected.

\section{Discussion}

In this work, we define emergence as 'a computational process of retrieving order from bifurcation and deterministic chaos embedded in equations governing coupled feedback' and life as 'a self-organizing, self-regulating entropy-maximizing iterator composed of a hierarchy of stable dissipative structures' (Appendix B). The conceptual model presented in Fig. 5 is emergent, as blockchain-based distributed systems employ bifurcation for self-regulation and artificial neural network (ANN) based AI performs self-organization as discussed below. It also fulfils the above definition of life. The ease with which the model 'organism' fits into existing systems of biological classification (Tables 2-4) suggest that artificial (engineered) dissipative structures, hierarchically arranged, duplicate biological organization and provide appropriate conditions for higher-level emergence to occur.

A critical component of the model shown in Fig. 5 is ANN-based artificial intelligence, which would be responsible for autonomous mining, transportation, production, repair, and system management. ANN-based AI forms the foundation of the rapidly growing field of machine learning, and ANN-based products are already in widespread commercial use, with applications including virtual assistants and autonomous driving. 
Fig. 6 Bifurcation and chaos in natural and artificial neural networks. a Neuron-to-neuron coupling generating regular and chaotic oscillations in model brain astrocytes. Peak amplitudes of calcium spikes $\left(q_{2}\right)$ in a model neuron are plotted against coupling strength $\left(k_{I P 3}\right)$. From Matrosov and Kazantsev (2011). b Bifurcation and chaos generated by two artificial neurons in coupled feedback. Plot shows relative signal at the inhibitory neuron $x$ as a function of neuron gain mu. From Wang (1991). c Model for human memory based on the chaotic dynamics of an artificial neural network (ANN). $W_{2}$ represents relative concentration of an inhibitory neurotransmitter. From Aram et al. (2017)
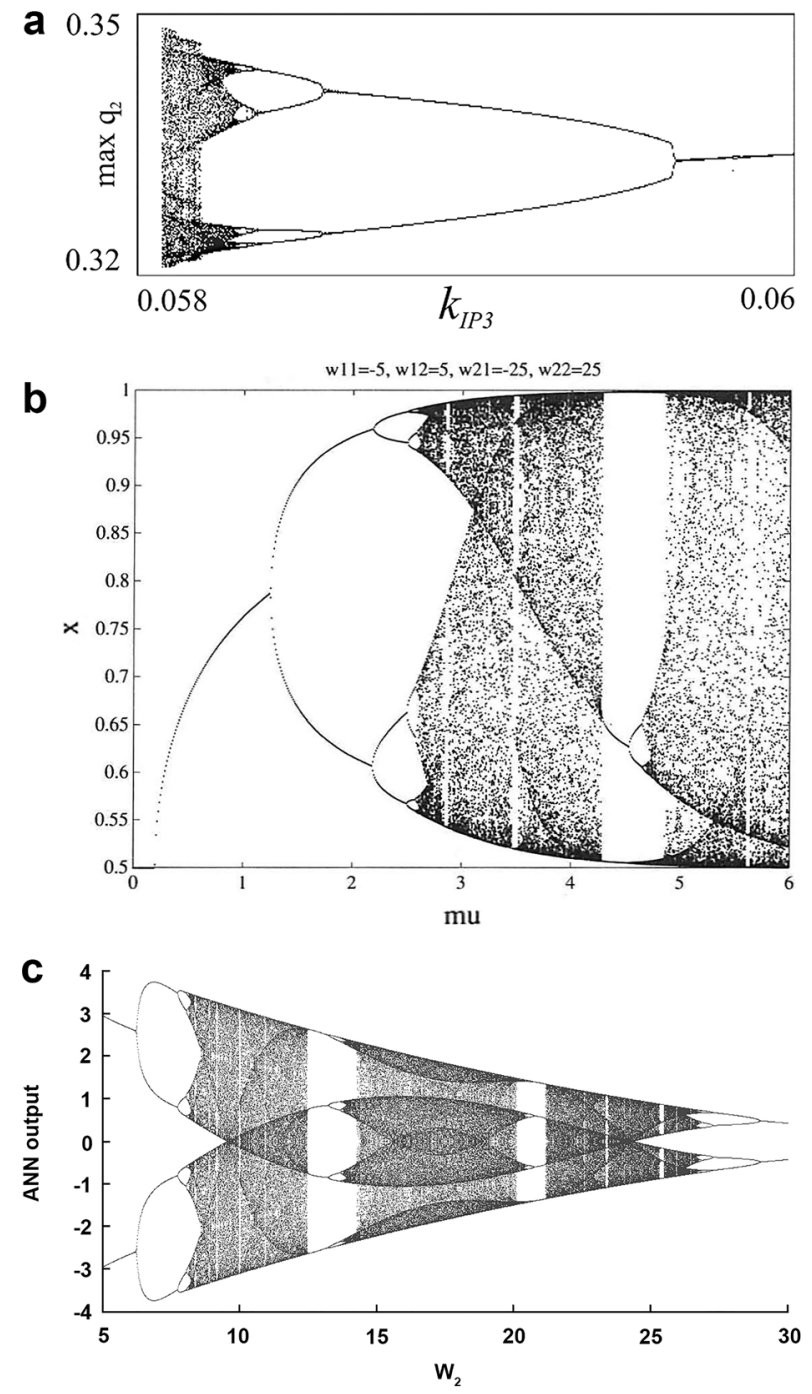

It is in many respects modeled on the workings of the brain, including coupled feedback between artificial neurons in more advanced architectures, such as recurrent neural networks (e.g., Pineda 1987). It has been mathematically proven that a simple neural network of only two neurons, one excitatory and the other inhibitory, can generate bifurcation and chaos (Wang 1991), indicating that such a system is unambiguously emergent. Conversely, ANNs can model processes in the human brain, from calcium spikes in individual neurons to storing and recall of memories (Fig. 6). These neural networks are designed and trained rather than algorithmically programmed. As with the human brain, the exact mechanisms are often not fully understood, but in both cases, deterministic chaos can be a powerful mechanism for retrieval, storage, and processing of information (e.g., Aram et al. 2017). 
With the advent of the blockchain, ANN-based AI has potentially gained both an immutable storage medium and the processing capability of blockchain-based virtual machines. Integrating blockchain and ANN-based AI agents is currently an active area of research and development (e.g., Dinh and Thai 2018; Zhang et al. 2018; Salah et al. 2019; Krittanawong 2020). Several mechanisms have been proposed, including: (i) storing entire artificial neural nets on-chain, (ii) storing artificial neural nets on computers comprising a blockchain's virtual machine, or (iii) storing the neural net in cloud-based services. In the latter two cases, the neural net's hash would be written to the blockchain for positive verification. The distributed, global nature of blockchain-based dVMs, together with the immutability of their genetic carrier and the combined computational power of a large number of nodes may provide a fertile environment for the collaborative development and rapid prototyping of AI codes and neural nets.

ANN-based AI enabled recent advances in autonomous mining (e.g., Li and Zhan 2018; Hirayama et al. 2019), smart manufacturing (e.g., Kusiak 2018; Wang et al. 2018), autonomous driving (e.g., Li et al., 2018; Elluswamy et al. 2020; Talpes et al. 2020), and in conjunction with global, satellite-based internet (e.g., Su et al. 2019) make it possible in the near future to close the loop and enable blockchain-based organisms that are fully self-sustaining, self-organizing, self-regulating, self-repairing and self-reproducing (Fig. 5) entirely independently of any human intervention.

\section{Conclusions}

We present evidence that a blockchain shares a large number of functional and structural similarities with DNA, a chemistry-based data structure that provides fundamental instructions for all known forms of life. We demonstrate that blockchain-based distributed systems possess specific characteristics associated with life, such as growth and change, responses to environment, homeostasis, reproduction, and genetics through a series of reproducible observations conducted on public blockchain-based dVMs. We present a model of a simple decentralized, self-sustaining, self-organizing, self-regulating blockchain-based organism as an operationally closed system, and describe developing technologies that could enable it in the near future. Such systems would have a number of specific advantages over biological life, including significantly improved speed, accuracy, redundancy, and unlimited size of blockchain as opposed to DNA, the ability to pass acquired traits to offspring, the ability of specialized cells to operate independently of host organism, and a lack of programmed senescence and death. Public blockchain-based dVMs provide an uncontained environment for the development of ANN-based artificial general intelligence (AGI), potentially leading to an ability to direct their own evolution, resulting in rapid self-improvements and the potential to exceed human intelligence (e.g., Grace et al. 2018). 


\section{Appendix A - Bifurcation and Chaos}

\section{Logistic equation}

Li and Yorke (2004) and May (1975) used the term 'chaos' to describe 'non-periodic' solutions unstable with respect to small modifications, so that slightly differing initial states can evolve into considerably different states' (Lorenz, 1963), noting that chaos can be generated by iterating the logistic equation:

$$
x_{n+1}=r x_{n}\left(1-x_{n}\right)
$$

where $r$ represents positive feedback (e.g., growth rate) on $x$ (e.g., population) as $n$ is incremented, and (1-x) represents negative feedback (e.g., environmental constraints) on $x$. The logistic equation is applicable to a broad range of systems with coupled positive and negative feedback, such as biological populations in a predator-prey system (e.g., May, 1975; Cushing, 1977). As the equation is iterated, $x_{n}$ stabilizes at a constant, oscillates between two or more values, or varies in an irregular pattern exhibiting fractal self-similarity (Fig. 7), depending on the value of $r$.

\section{Bifurcation diagram}

This behavior can be generalized using a bifurcation diagram (Fig. 8), which plots the results of iterating the logistic equation over a range of $r$ values (e.g., May, 1976). This bifurcation phenomenon is generic to a broad spectrum of functions and systems of equations describing coupled feedback systems (e.g., May, 1976). Bifurcation and chaos has since been observed in a variety of real-world systems involving iteration of feedback loops (Fig. 9), such as visual (e.g., Crevier and Meister, 1998), cardiovascular (e.g., Garfinkel et al., 1992), and nervous (e.g., Schiff et al., 1994) systems of animals, as well as simple electronic circuits (e.g., Chua and Huynh, 1992) and artificial neural networks (e.g., Van der Maas et al., 1990).

Indeed, it provides the missing mathematical link to earlier work on an even broader variety of systems, now understood to be examples of chaos. The $n$-body problem, which describes gravitational interactions of $n$ celestial objects is one such example; for $n>2$, the objects undergo coupled feedback resulting an infinite number of solutions sensitively dependent on initial conditions (Poincaré, 1890). Einstein (1917), generalizing this result, defined type a and type b motion, with (a) integrable, with a solution predicted by the laws of motion, and (b) non-integrable, 'degenerate', having an infinite number of solutions. Lorenz (1963) described a computer-assisted study of solutions to systems of differential equations designed for weather prediction, reporting that 'for those systems with bounded solutions, it is found that non-periodic solutions are ordinarily unstable with respect to small modifications, so that slightly differing initial states can evolve into considerably different states.'

\section{Mandelbrot set}

Iterating the logistic equation with complex $r$ and $x$ generates an extensive fractal (Mandelbrot 1980; 1983), now generalized into what is termed the Mandelbrot set (Brooks and Matelski, 1981; Douady and Hubbard, 1984). The Mandelbrot set $\mathscr{M}$ can be visualized 

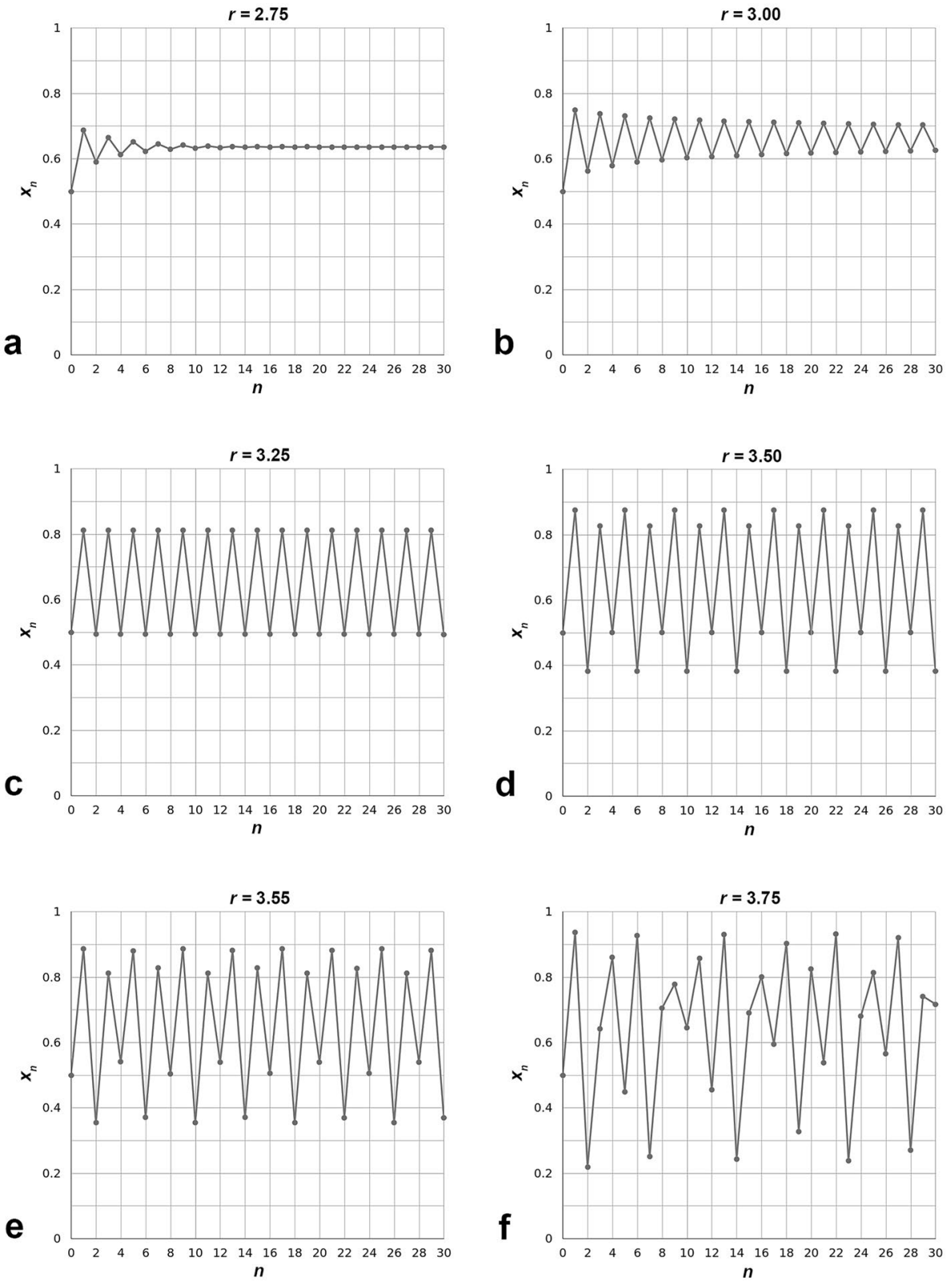

Fig. 7 Iteration of the logistic equation $x_{n+1}=r x_{n}\left(1-x_{n}\right)$ for increasing values of $r$, with initial population $x_{0}$ set to 0.5 . a) $r=2.75, x_{n}$ stabilizes a constant value. b) $r=3 ; x_{n}$ stabilizes to a periodic cycle between two values. c) $r=3.25 ; x_{n}$ in a periodic cycle between two values. d) $r=3.50 ; x_{n}$ in a periodic cycle between four values. e) $r=3.50 ; x_{n}$ in a periodic cycle between eight values. f) $r=3.75 ; x_{n}$ in a chaotic regime exhibiting fractal self-similarity 

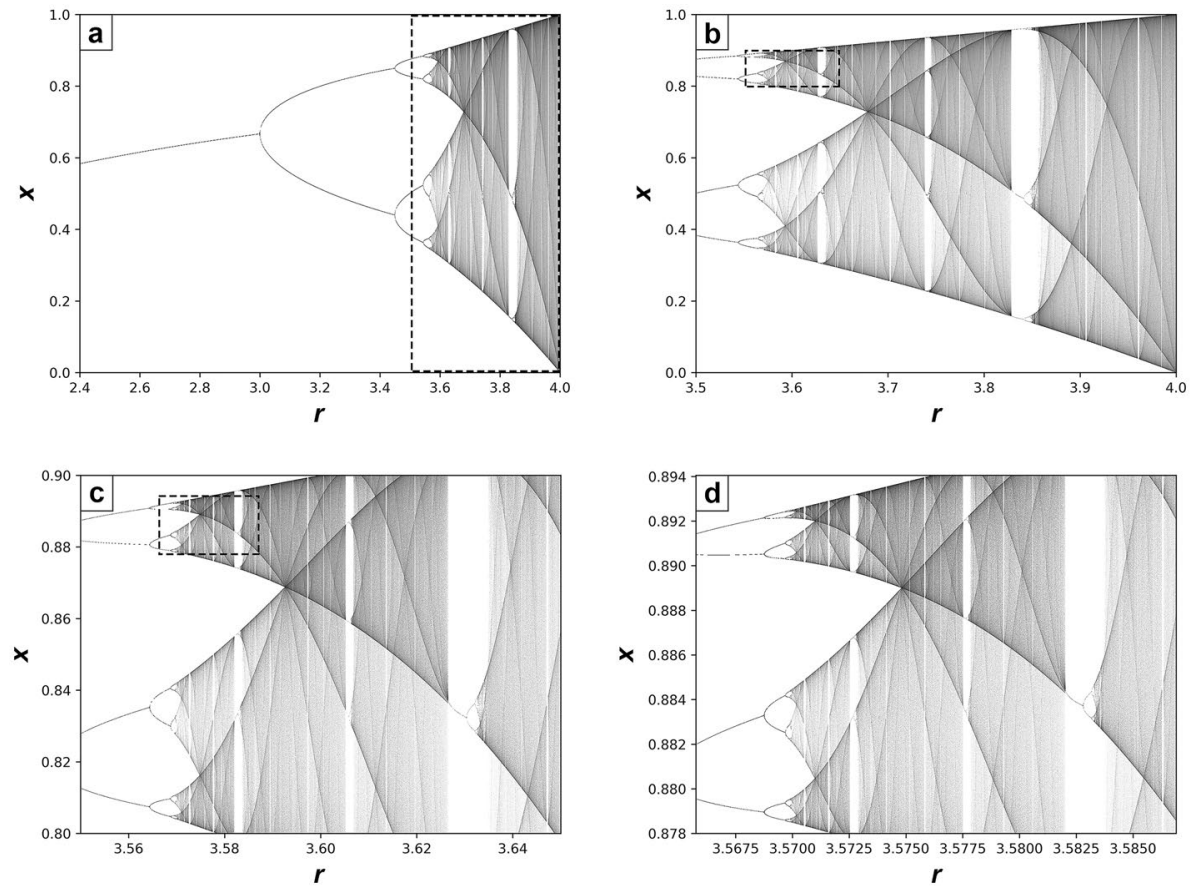

Fig. 8 a) Bifurcation diagram obtained by $10^{5}$ iterations of the logistic equation $x_{n+1}=r x_{n}\left(1-x_{n}\right)$. Note the symmetry-breaking bifurcations starting at $r=3$, and the sudden appearance of chaos at a critical value of $r \approx 3.57$. Dashed box represents the area shown in the next panel. b) Chaotic region between $r \approx 3.57$ and $r=4$. Note the interspersed regions of chaos (grey areas) and stability. c-d) Self-similarity at different spatial scales, a defining characteristic of fractals. For this and other figures, source code is given in Supplementary Information
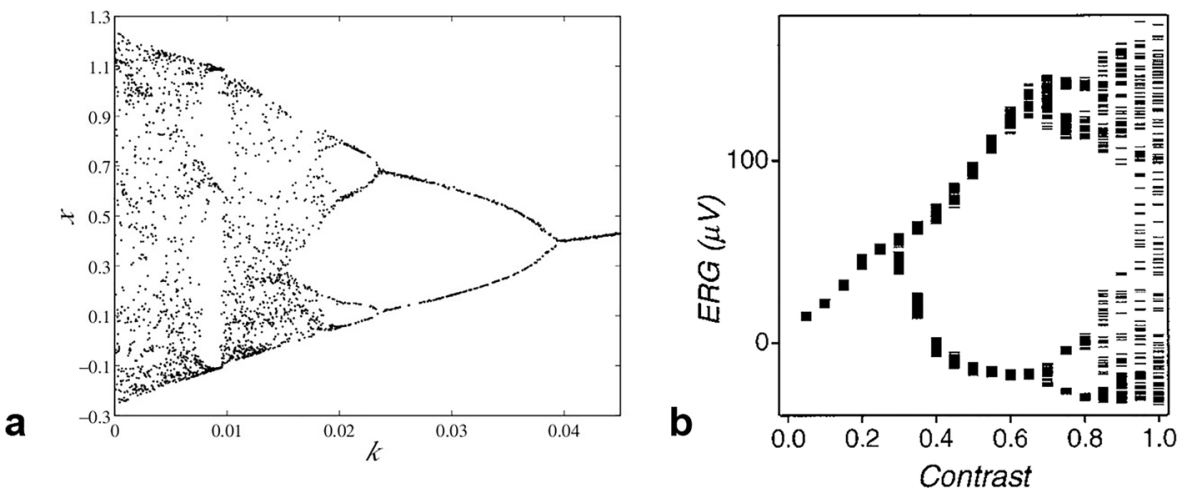

Fig. 9 Examples of observed bifurcation and chaos in coupled feedback systems. a) Bifurcation diagram for a perturbed Chua's circuit, where $k$ is the amplitude of the perturbation and $x$ is capacitor voltage. From Tereshko (2011). b) Bifurcation diagram for the electrical response of salamander retinal cells to light flashes of varying contrast. From Crevier and Meister (1998) 


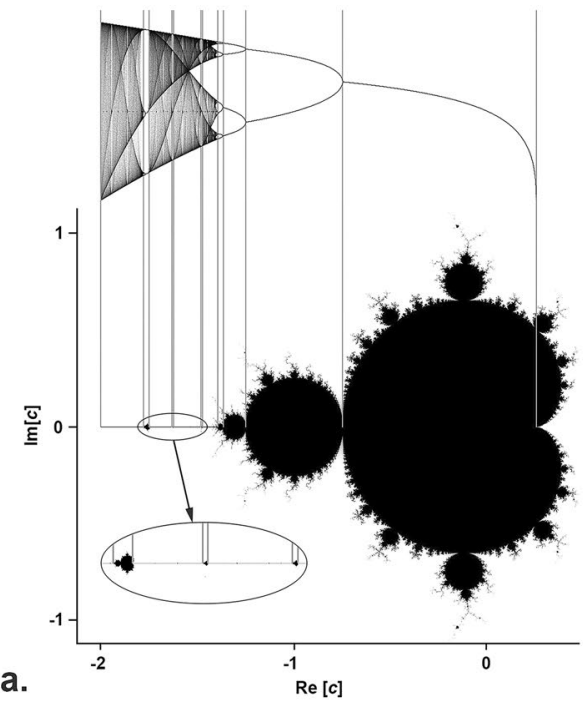

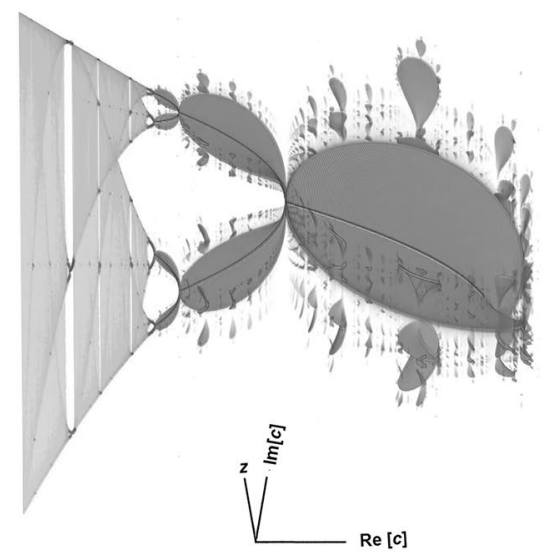

b.

Fig. 10 Relationship between the bifurcation diagram obtained by iterating the logistic equation $x_{n+1}=r x_{n}\left(1-x_{n}\right)$, and the Mandelbrot set, obtained by iterating the equation $z_{n+1}=z_{n}^{2}+c$, where $z$ and $c$ are complex numbers. a) Features on the logistic map correspond to those on the real $(\operatorname{Re}[c])$ axis of the Mandelbrot set. The chaotic region of the logistic map corresponds to the needle of the Mandelbrot set. Note that the regions of stability correspond to 'minibrots'. The large circle centered on $[-1,0]$, can be exactly fitted with the equation $c=1 / 4 e^{i \theta}-1$, a form similar to Euler's identity. b) The Mandelbrot set and the logistic map rotated $45^{\circ}$ around the $\mathrm{x}$-axis

by iterating the equation $z_{n+1}=z_{n}^{2}+c$ where $c$ and $z$ are complex numbers, and plotting points that do not escape to infinity (Fig. 10). The boundary of $\mathscr{M}$ is chaotic; part of this chaos is sampled by the logistic equation in the needle of $\mathscr{M}$ (between $c \approx-1.40$ and $c=$ -2 along the real axis), and other parts are likewise computable with real numbers (e.g., Hertling, 2005; Braverman and Yampolsky, 2008) using iterated feedback systems.

\section{Fractals and chaos}

Figure 11 provides examples of a wide range of fractal structures found at the boundary of $\mathscr{M}$, illustrating that chaos is inherently fractal (e.g., Turcotte, 1997; Mandelbrot, 2004). Fractals are self-similar geometric structures common in nature (e.g., Mandelbrot, 1983; Turcotte, 1997), for example, coastlines, turbulent flows, snowflakes, clouds, trees, and capillaries exhibit fractal geometry (e.g., Mandelbrot, 1983; Turcotte, 1997). Chaos is also inherently low entropy (highly ordered), as illustrated by: (i) the low amount of information needed to generate the images in Fig. 11, consisting only of the equation $z_{n+1}=z_{n}^{2}+c$, and 2D Cartesian (xy) coordinates, (ii) numerous examples of geometric symmetry typically associated with a high degree of order and organization and (iii) the demonstrated use of fractals in image compression, effectively substituting bitmap image components with matching fractals (e.g., Jacquin, 1992). As such, chaos in this context is essentially the opposite of its modern vernacular definition ('a state of extreme disorder'). The term 'deterministic chaos' is often used in the context of chaos theory, and is adopted in this work. 


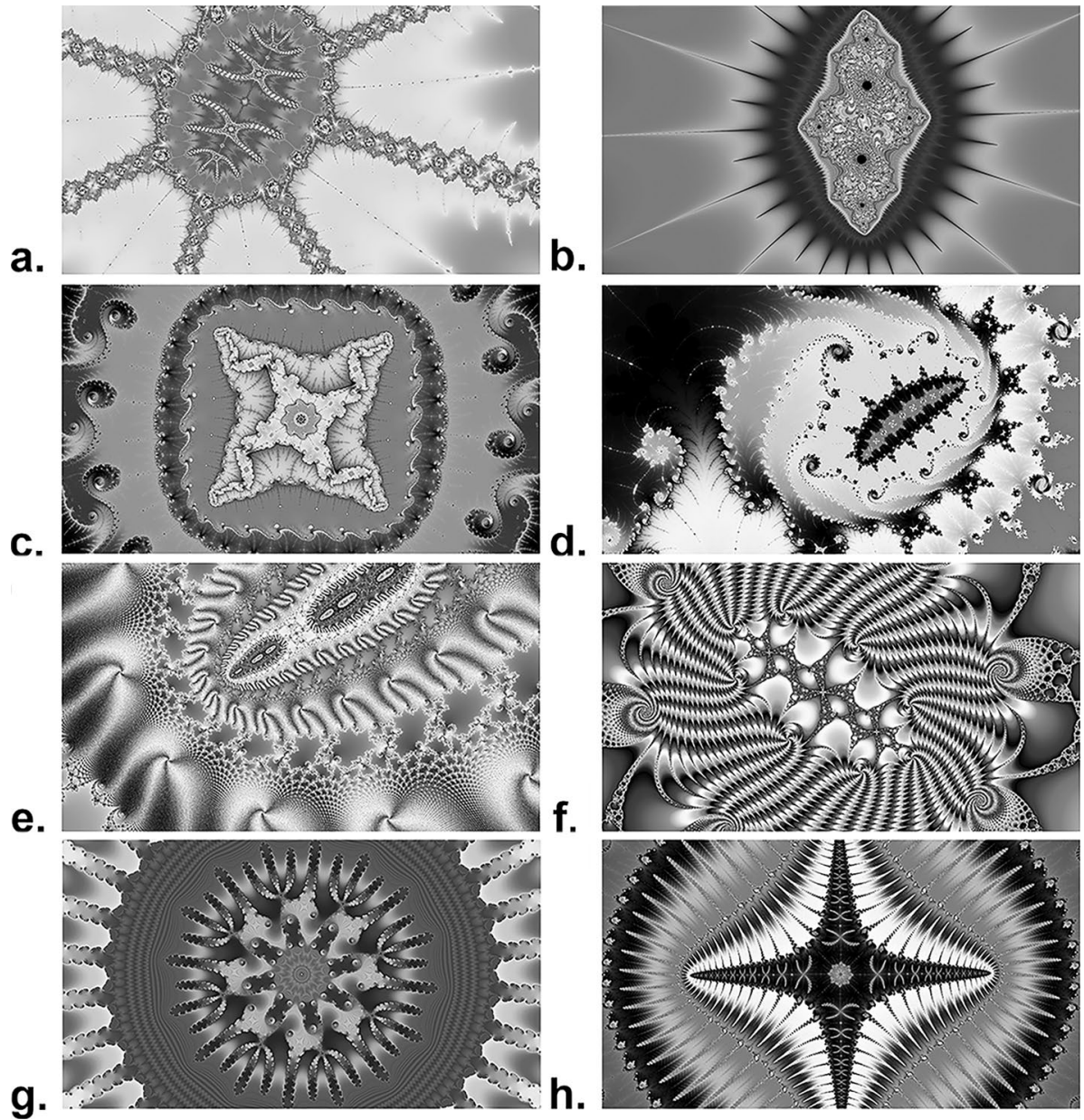

Fig. 11 (a-h) Example structures at the boundary of the Mandelbrot set, sampling chaos. Obtained by iterating the equation $z_{n+1}=z_{n}^{2}+c$, where $z$ and $c$ are complex numbers. Coordinates for each image are given in Supplementary Information. Note the apparently high degree of order and organization, and a general similarity to biological structures, with common motifs of cells, chromosomes, spikes, spirals, etc.

\section{Self-organization}

From the above, it appears a reasonable working hypothesis that the order used for at least some of the self-organization observed in the natural world, particularly in biology, is embedded in equations governing coupled feedback, and is retrieved through their iteration. This potentially explains observations of the Fibonacci sequence (in which each number is the sum of the two preceding ones), which is ubiquitous in the chaotic boundary of $\mathscr{M}$, in biological systems (e.g., Newell and Shipman, 2005; Yamagishi and Shimabukuro, 2008). The process of self-organization via the retrieval of order from bifurcation and deterministic chaos is termed emergence, and is discussed in detail in Appendix B. 


\section{Appendix B - Emergence and Life}

Modern definitions of emergence include:

- Emergence is generally understood to be a process that leads to the appearance of structure not directly described by the defining constraints and instantaneous forces that control a system' (Crutchfield 1994).

- 'Common properties that identify [phenomena] as emergent: (1) radical novelty (not previously observed in complex systems); (2) coherence or correlation (components maintain identity); (3) A global or macro level (components form a novel whole); (4) it is the product of a dynamical process (associated with bifurcation); and (5) it is ostensive (can be perceived)' (Goldstein, 1999).

- 'A system exhibits emergence when there are coherent emergents at the macro-level that dynamically arise from the interactions between the parts at the micro-level. Such emergents are novel w.r.t. the individual parts of the system' (De Wolf and Holvoet, 2004).

We define emergence as an iterative computational process of retrieving order from bifurcation and deterministic chaos embedded in equations governing coupled feedback. The requirements for emergence are (i) coupled feedback between system components and (ii) energy to drive iteration within the system. The process of emergence provides an explanation for, and is fully consistent with, the appearance of highly ordered (low entropy) thermodynamic dissipative structures (e.g., Prigogine and Nicolis, 1967; Prigogine and Lefever, 1968) when a dynamical system is pushed far from equilibrium by the input of energy. Under this paradigm, emergence begins at the first bifurcation point (Figs. 7 and 8), equivalent to the thermodynamic 'critical point of instability', when the system can no longer reach equilibrium regardless of the number of iterations.

Given the deterministic nature of this process, the development of a general mathematical theory of emergence should be possible and is a promising direction for future work. Here, we give several examples and describe how the definition above applies to each case.

\section{Belousov-Zhabotinsky (BZ) reaction}

The BZ reaction (Belousov 1959; Zhabotinsky 1964) is a commonly cited (e.g., Rössler and Wegmann, 1978; Agladze et al., 1984; Richetti et al., 1987) example of bifurcation and deterministic chaos. It is an exothermal chemical reaction in which malonic acid is oxidized by bromate, catalyzed by a metal ion such as ferroin. In the course of the reaction, intermediates $\left(\mathrm{Br}^{-} \mathrm{Br}_{2}, \mathrm{HOBr}, \mathrm{HBrO}_{2}, \mathrm{BrO}_{2}\right)$ form a coupled feedback system, which exhibits periodic, quasi-periodic, or 'chaotic' behavior depending on parameters, mapped out in the bifurcation diagram (Fig. 12). The energy is provided by the exothermal nature of the reaction. This system is a model for various types of biophysical communication networks based on encapsulated oscillating reactions (e.g., Tyson et al., 1989; Torbensen et al., 2015; 2017). The BZ reaction can serve as a chemical neuron that can process both Boolean and fuzzy logic (e.g., Holley et al., 2011; Gentili et al., 2012). 

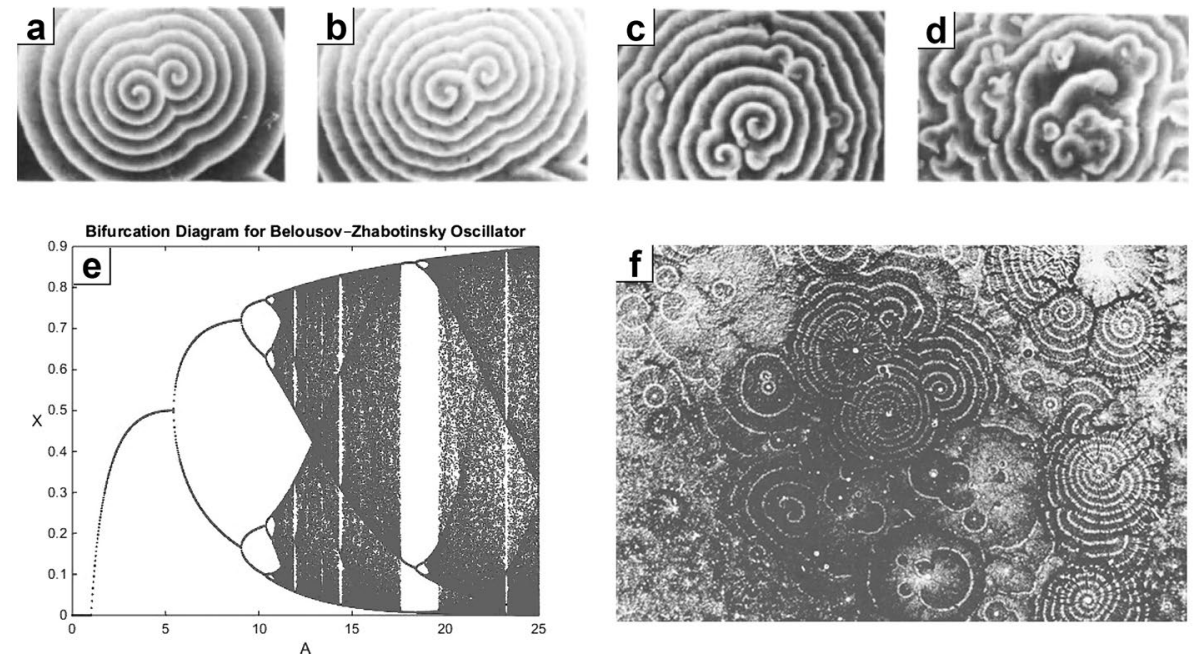

Fig. 12 (a-d) Belousov-Zhabotinsky (BZ) reaction in a 9-cm petri dish. a. 5 min after mixing of reagents. Waves moving from the center to the periphery are observed, with a spiral pattern in the center. b. 6 min. Waves assume sinusoidal form and notches appear. c. $10 \mathrm{~min}$. Wave breaks curl into rotating spirals. d. 11 min. Formation of fragments and small-scale turbulence. Modified from Agladze et al. (1984). e. Bifurcation diagram calculated for the BZ reaction. $X$ is relative concentration of one of $\mathrm{HBrO}_{2}$, an active oscillating intermediate reagent, $A$ is relative reaction rate. From Eskew and Simonyi (2017). f. Biological analog: Chemical communication in slime mold Dictyostelium discoideum. Oscillating pacemaker cells initiate waves of cAMP. From Tyson et al. (1989)

\section{Hexagonal lattice}

The hexagonal lattice is a bifurcation-based emergent structure occurring in a broad range of biological and abiological contexts:

- Rayleigh-Bénard convection cells (Fig. 13a). The energy source is the heated bottom boundary. The iteration cycle consists of the warmer fluid reaching the upper boundary, losing some of its heat, and sinking back down to the bottom. The iterated coupled feedback between upwelling and downwelling fluid results in bifurcation (e.g., Ma and Wang, 2004; Venturi et al., 2010) and formation of geometrically regular, typically hexagonal, convection cells.

- Faraday waves (Fig. 13b). The energy source is the externally applied vibrations, which result in alternating compression and relaxation cycles within the liquid, forming coupled positive and negative feedback. The iteration cycle corresponds to the vibration frequency, typically on the order of $20 \mathrm{~Hz}$. The iterated coupled feedback results in bifurcation (Douady and Fauve, 1988; Wagner et al., 1999), and formation of geometrically regular, such as hexagonal, standing waves on the surface of the liquid.

- A frequently reported (e.g., Klüver, 1928) human visual hallucination pattern (Fig. 13c). It can be modeled with an artificial neural net (Ermentrout and Cowan, 1979), and generally understood to be a bifurcation-based product of coupled feedback between neurons in the human visual cortex (e.g., Bressloff et al., 2001; 2002). The iteration cycle corresponds to the frequency of synchronized neuron firing in the visual 

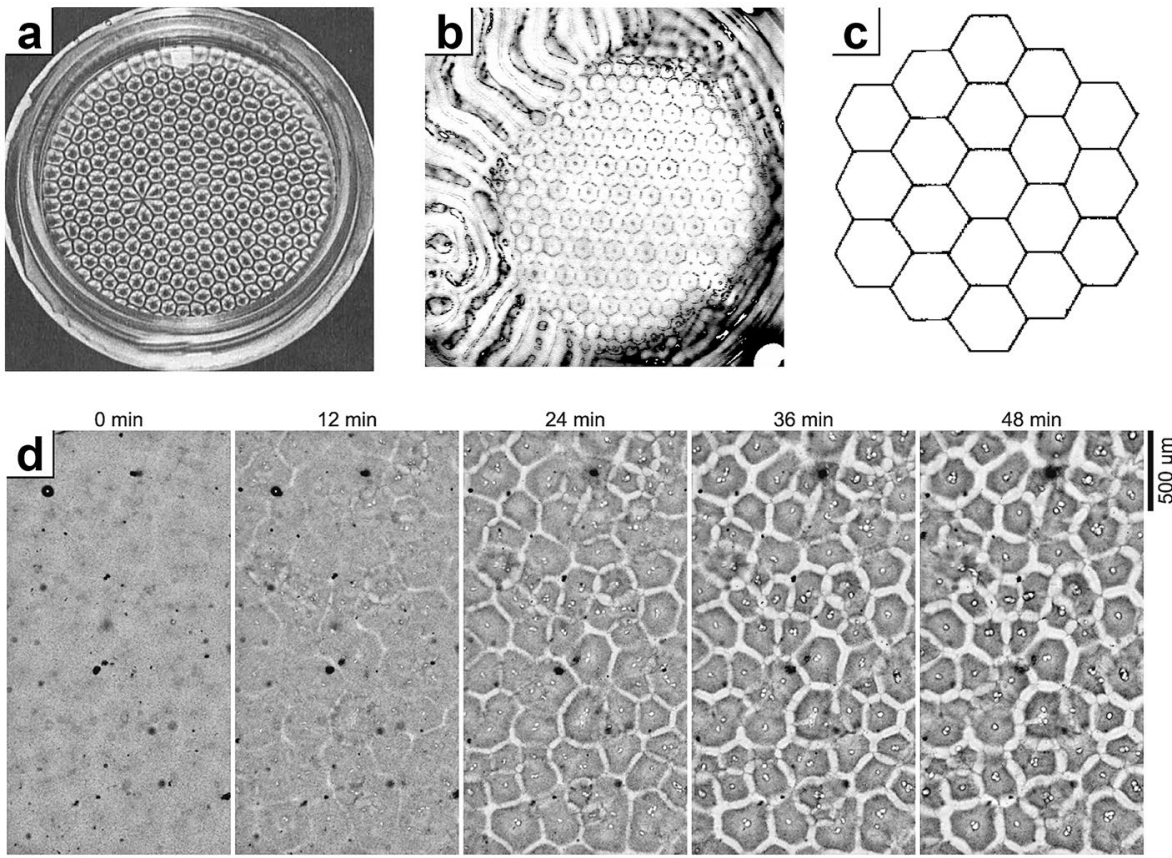

Fig. 13 a. Rayleigh-Bénard convection cells in silicone oil in a 10-cm plane plate heated from below. From Koschmieder (1974). b. Faraday waves in silicone oil in a 32-cm circular container vibrating at a frequency of $20 \mathrm{~Hz}$. From Kudrolli and Gollub (1996). c. Common human visual hallucination pattern calculated using coupled feedback in a two-layer artificial neural net. From Ermentrout and Cowan (1979). d. Cell self-assembly from molecular interactions in homogenized egg cytoplasm from African clawed frog, Xenopus laevis. From Cheng and Ferrell (2019)

cortex, on the order of $10 \mathrm{~Hz}$ (Henke et al., 2009). The energy source is the stored electrical potential within neurons.

- Cell self-assembly (Fig. 13d). Reported by Cheng and Ferrell (2019) in homogenized egg cytoplasm from African clawed frog. Newly formed cells are observed to form a hexagonal lattice. No genetic material is present. In the context of emergence, the formation of this pattern would be expected to arise from coupled feedback between molecular components of the cytoplasm. The energy source is adenosine triphosphate (ATP). Unlike previous examples, a stable physical structure is formed.

- Honeycomb formation. It has been mathematically proven (Hales, 2001) that a hexagonal lattice is optimal in terms of dividing a surface into regions of equal area with the least total perimeter, which explains why it was evolutionarily retained. However, a mathematical model for honeycomb formation has not yet been published. Emergence theory predicts that a honeycomb is a bifurcation-based emergent structure formed as a result of coupled feedback between worker bees. 

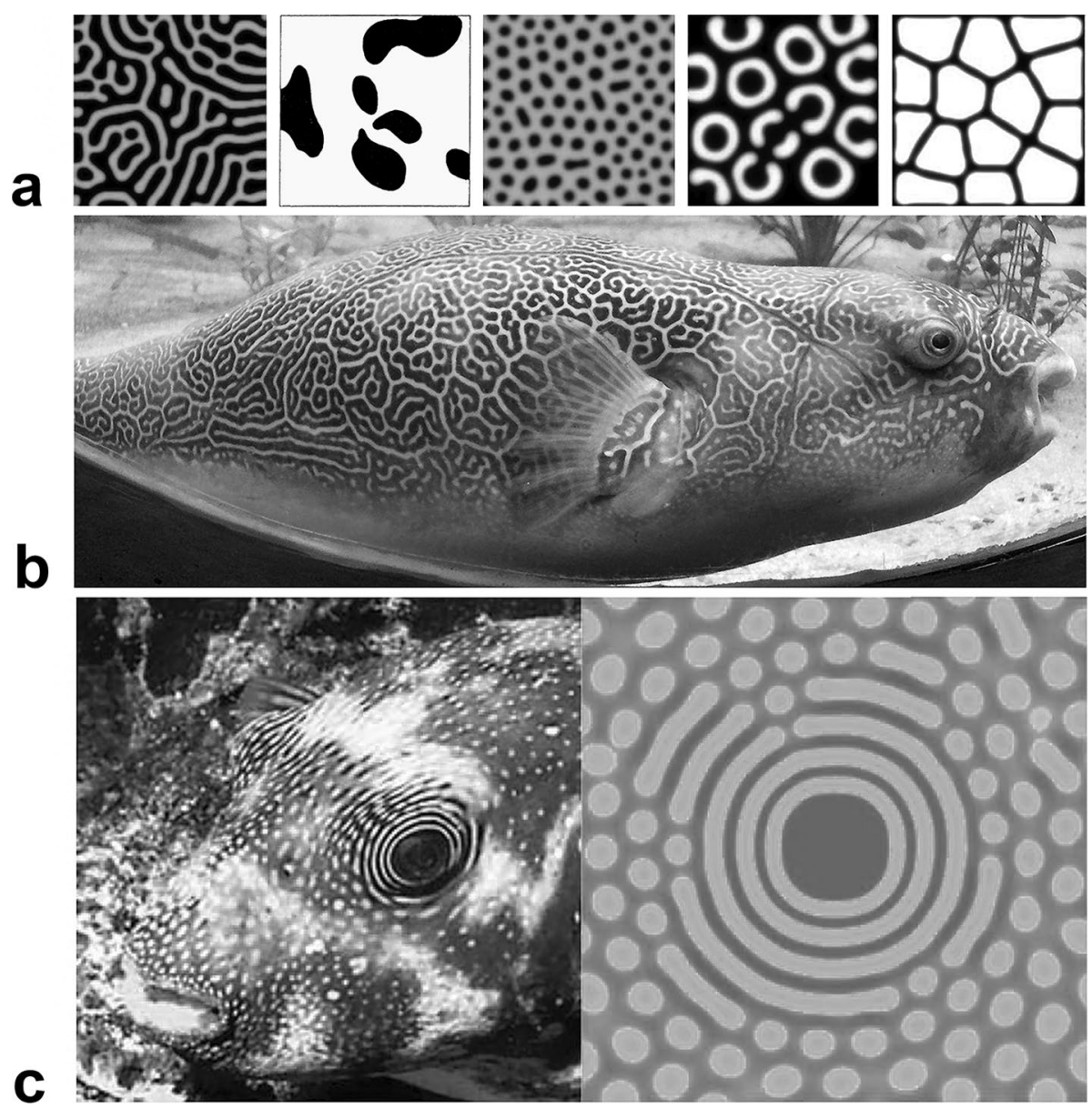

Fig. 14 Turing patterns, morphogenesis. a. Examples of calculated Turing patterns. b. Mbu pufferfish (Tetraodon $\mathrm{mbu}$ ) exhibiting a Turing pattern. c. Complex pattern around the eye of white-spotted pufferfish (Arothron hispidus, left) simulated using Turing pattern mechanisms (right). Modified from Turing (1952) and Kondo and Miura (2010)

\section{Turing patterns}

Turing (1952) proposed a theory of mophogenesis based on a dynamical system involving coupled-feedback reactions and diffusion in which bifurcation generates spatially nonuniform stationary solutions (e.g., Murray, 1990; Forgacs and Newman, 2005). It has been remarkably successful at reproducing patterns observed in animals (Fig. 14) and continues to be the leading explanation for how genes (and the proteins they encode) produce observed morphologies (e.g., Murray, 1990; Tompkins et al., 2014). The core of the Turing (1952) morphogenesis theory shares a number of similarities with the later-discovered BZ reaction in that the components form a coupled feedback system that produces ordered patterns using a mechanism based on bifurcation and deterministic chaos (e.g., Rössler, 1976; Tompkins et al., 2014). 

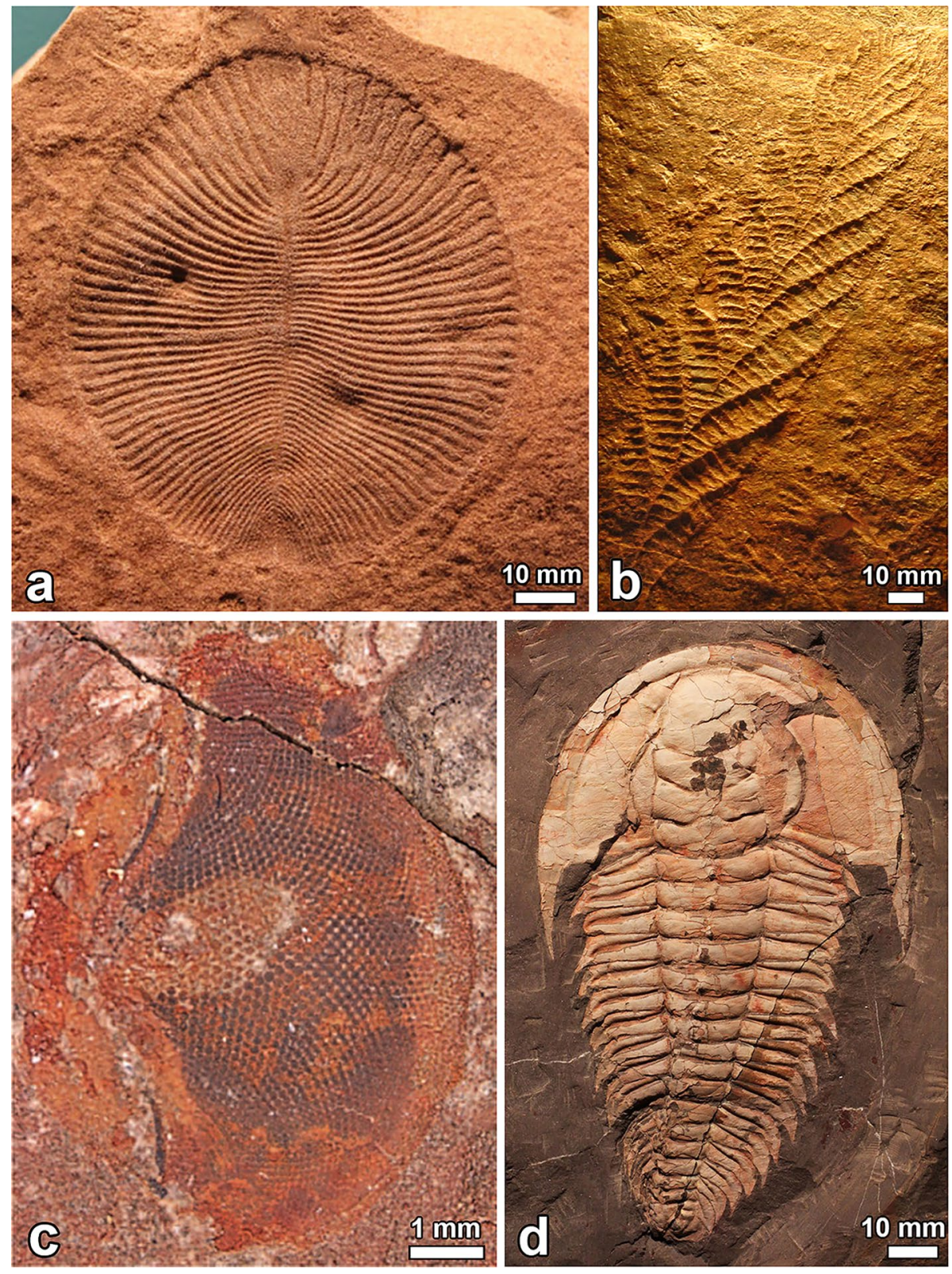

Fig. 15 Multicellular emergence as a possible cause of Cambrian explosion. a. Late Ediacaran fossil of genus Dickensonia. From Hoekzema et al. (2017). b. Late Ediacaran fossil of genus Charnia. From Hoekzema (2015). c. Early Cambrian fossil arthropod eye with hexagonal pattern typical for emergent structures. From Lee et al. (2011). d. Early Cambrian fossil trilobite Redlichia rex. From Holmes et al. (2019) 

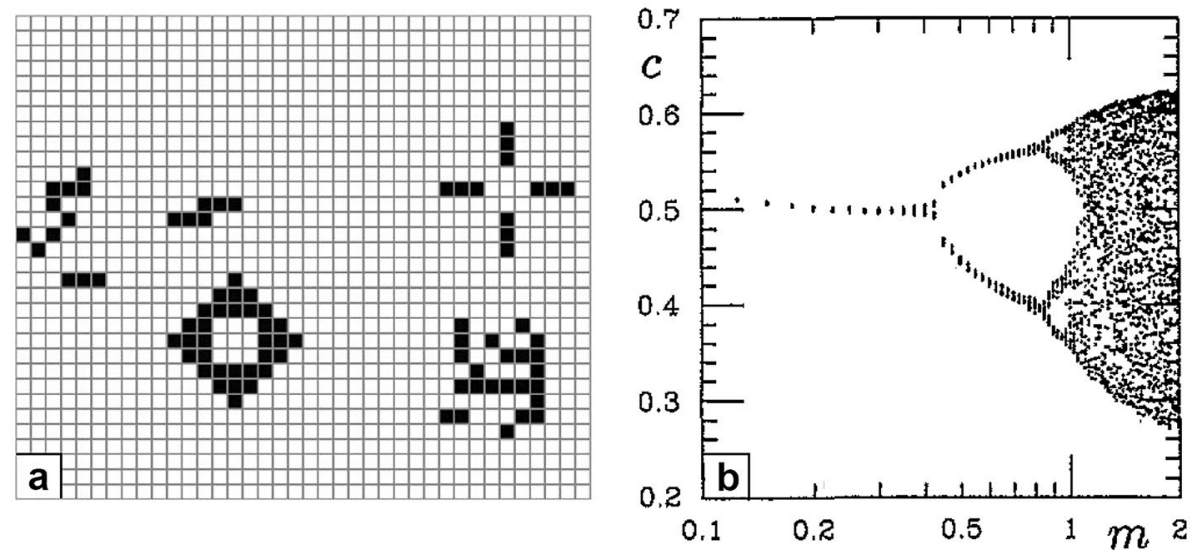

Fig. 16 Self-organization of cellular automata. a. Example of patterns produced by the Game of Life after 100 steps (generations) starting from a random array of equally likely 0 s and $1 \mathrm{~s}$ of size $10 \times 15$. From Zenil (2015). b. Bifurcation diagram for a Game of Life type cellular automata network. $m$ is a 'mixing parameter' determining the distance a cell is allowed to move each iteration, and $c$ is relative density of non-zero cells. From Boccara et al. 1994

\section{Cambrian Explosion}

Arguably one of the most significant weaknesses in the theory of evolution is the so-called 'Darwin's dilemma', 'the manner in which species belonging to several of the main divisions of the animal kingdom suddenly appear in the lowest known [Cambrian-age] fossiliferous rocks' (Darwin, 1859). This was later termed the 'Cambrian explosion', referring to the sudden appearance of nearly all major animal phyla in a geologically short time period between 542 and 520 million years ago (e.g., Zhuravlev and Riding, 2001), the cause of which is not well understood (e.g., Marshall, 2006). The roots of the Cambrian explosion extend 30 million years earlier to the period termed the Avalon explosion (Shen et al., 2008; Xiao and Laflamme, 2009) in the Late Ediacaran period, referring to the appearance and proliferation of new types of animals exhibiting simple geometric organization and bilateral symmetry. Perhaps the earliest of these is the genus Dickinsonia (e.g., Bobrovskiy et al., 2018).

The appearance of Dickinsonia (Fig. 15a) may be explained by bifurcation-based emergence on multicellular level. Although multicellular animals (metazoans) such as Otavia antiqua appeared significantly earlier in the fossil record, between 720 and 660 million years ago (e.g., Valentine, 2002), these earlier organisms were morphologically amorphous. Dickinsonia, in contrast, consists of segments that increase in number as the organism grows. The geometric regularity of Dickinsonia can be explained by a growth model (e.g., Hoekzema, 2015; 2017) incorporating a mechanism similar to the BZ reaction, oscillating between two states as the organism grows, resulting in a regular pattern of segments.

Another common animal fossil, of genus Charnia (Fig. 15b), also appeared in the Late Ediacaran and repeats this pattern, but in addition to primary alternating segments there are secondary segments, approximately equally spaced, perpendicular to primary segments. This self-similarity gives it a fractal order of two (Hoekzema, 2015).

The beginning of the Cambrian period is marked by a significant increase in morphological diversity built on repeating geometric patterns. One of these is the hexagonal 
lattice, a common bifurcation-based emergent structure discussed earlier, observed in the fossilized eye from the early Cambrian (Fig. 15c).

This evidence suggests that a possible cause of the Cambrian explosion was emergence, or life evolving the ability to self-organize on the multicellular level using order obtained from bifurcation and deterministic chaos. This self-organization resulted in a rapid rise in complexity (e.g., Sepkoski, 1979), as illustrated by the complex geometry of trilobites (Fig. 15d) from the early Cambrian.

\section{Cellular automata}

The computational essence of emergence implies that it can be generated with computers, and indeed, this is the case. An example is cellular automata, such as the Game of Life (Conway, 1970; Gardner, 1970), which produce complex ordered patterns, and even organized systems, from arbitrary initial conditions and simple rules. The rules set up a system of coupled positive and negative feedbacks, and are iterated, fulfilling the requirements for emergence to occur and leading to observed bifurcation and deterministic chaos (e.g., Wolfram, 1984; Nowak and May, 1992; Boccara et al., 1994). Figure 16 shows selforganization of cellular automata from disordered (high entropy) initial states into ordered (low entropy) geometric patterns. Like the BZ reaction, cellular automata can be used to implement basic logical operations (e.g., Rennard, 2002), and, taking it a step further, construct a virtual universal Turing machine (e.g., Rendell, 2002) capable of performing any computer operation (Turing, 1936), illustrating how iterative application of simple rules involving coupled feedback can create extremely complex systems.

\section{Definition of life}

As discussed in the main body of the paper, life has been difficult to define precisely. Based on the concept of emergence, we propose the following definition: Life is a self-organizing, self-regulating entropy-maximizing iterator composed of a hierarchy of stable dissipative structures. 'Self-organizing' refers to retrieving order from bifurcation and deterministic chaos via 'self-regulating' coupled feedback, 'iterator' refers to feedback cycles, or iterations, 'entropy maximization' and 'dissipative structures' grounds the definition in wellestablished thermodynamic concepts, 'hierarchy' refers to lower-order building blocks producing higher-order building blocks, and 'stable' means that continuous external energy input is not required. This definition applies to the simplest organisms, composed of, at a minimum, spontaneously folded macromolecules (proteins (e.g., Böhm, 1991) and nucleic acids (e.g., Coleman et al., 2000)) and self-organized lipid bilayer membranes (e.g., Cheng and Ferrell, 2019), as well as complex organisms that also exhibit emergence on multicellular and multi-organism (e.g., bird flocks, fish schools, insect colonies, etc.) levels. It excludes common counter-examples, such as fire, storm systems, convective systems, and crystal growth.

Supplementary Information The online version contains supplementary material available at https://doi. org/10.1007/s11084-021-09608-1.

Acknowledgements O.A.'s contributions to this work were funded in part by the NASA Habitable Worlds program grant 80 NSSC17K0740. S.J.M. extends special thanks to the Erwin Schrödinger International Institute for Mathematical Physics (Vienna, Austria) for visiting support during the initial stages of this 
work, and the University of Vienna, Department of Lithospheric Research (Vienna, Austria) for the Ida Pfeiffer Guest Professorship at which time this manuscript was completed. A discussion on blockchain at PSI on February 5, 2018 organized by M. Sykes is acknowledged. O.A. is grateful for discussions with E. Chudakov and I. Gembitsky.

Authors' Contributions K.L.B. conceived the original idea, produced the conceptual framework, and outlined the theoretical flow. S.J.M. encouraged O.A. and K.L.B. to investigate definitions of life from mechanistic and informational aspects, and supervised the project. O.A. designed the computational framework and wrote the manuscript with input from K.L.B and S.J.M. All authors discussed the interpretation of results and contributed to the final manuscript.

Funding This work was funded in part by the NASA Habitable Worlds program grant 80NSSC17K0740.

Data Availability Data was obtained from a public blockchain and is by definition available and immutable.

Code Availability All source code needed to replicate our results is provided in the Supplementary Information and will be uploaded to Github.

\section{Declarations}

Conflicts of Interest Authors declare no conflicts of interest or competing interests.

Open Access This article is licensed under a Creative Commons Attribution 4.0 International License, which permits use, sharing, adaptation, distribution and reproduction in any medium or format, as long as you give appropriate credit to the original author(s) and the source, provide a link to the Creative Commons licence, and indicate if changes were made. The images or other third party material in this article are included in the article's Creative Commons licence, unless indicated otherwise in a credit line to the material. If material is not included in the article's Creative Commons licence and your intended use is not permitted by statutory regulation or exceeds the permitted use, you will need to obtain permission directly from the copyright holder. To view a copy of this licence, visit http://creativecommons.org/licenses/by/4.0/.

\section{References}

Agladze KI, Krinsky VI, Pertsov AM (1984) Chaos in the non-stirred Belousov Zhabotinsky reaction is induced by interaction of waves and stationary dissipative structures. Nature 308(5962):834-835

Alberts B, Johnson A, Lewis J, Raff M, Roberts K, Walter P (2002) Molecular biology of the cell. Garland Science, New York

Aram Z, Jafari S, Ma J, Sprott JC, Zendehrouh S, Pham VT (2017) Using chaotic artificial neural networks to model memory in the brain. Commun Nonlinear Sci Numer Simul 44:449-459

Aristotle (c. 330 BCE) Metaphysics, Book H (Eta), 1045, 8-10

Arrhenius G, Sales B, Mojzsis S, Lee T (1997) Entropy and charge in molecular evolution-the case of phosphate. J Theor Biol 187(4):503-522

Ault JT (2018) Advancing the science and impact of blockchain technology at Oak Ridge National Laboratory (No. ORNL/TM-2018/1026). Oak Ridge National Lab.(ORNL), Oak Ridge, TN (United States).

Back A(2002) Hashcash-a denial of service counter-measure. White paper, downloaded from http://www.hashcash. org/hashcash.pdf

Back A, Corallo M, Dashjr L, Friedenbach M, Maxwell G, Miller A, Wuille P (2014) Enabling blockchain innovations with pegged sidechains. http://www.opensciencereview.com/papers/123/enablingblockchaininnovations-with-pegged-sidechains, 72

Bahi JM, Couchot JF, Guyeux C, Salomon M (2012) Neural networks and chaos: Construction, evaluation of chaotic networks, and prediction of chaos with multilayer feedforward networks. Chaos 22(1):013122

Barnsley MF, Devaney RL, Mandelbrot BB, Peitgen HO, Saupe D, Voss RF, McGuire M (1988) The science of fractal images (pp. xiv-312). New York: Springer

Belousov BP (1959) An oscillating reaction and its mechanism. Sbornik referatov po radiatsionnoi meditsine, Medgiz, Moscow, 145-152

Bernstein DJ, Lange T (2017) Post-quantum cryptography. Nature 549(7671):188-194 
Bérut A, Arakelyan A, Petrosyan A, Ciliberto S, Dillenschneider R, Lutz E (2012) Experimental verification of Landauer's principle linking information and thermodynamics. Nature 483(7388):187-189

Bobrovskiy I, Hope JM, Ivantsov A, Nettersheim BJ, Hallmann C, Brocks JJ (2018) Ancient steroids establish the Ediacaran fossil Dickinsonia as one of the earliest animals. Science 361(6408):1246-1249

Boccara N, Roblin O, Roger M (1994) Route to chaos for a global variable of a two-dimensional 'game-of-life type' automata network. J Phys Math Gen 27(24):8039

Böhm G (1991) Protein folding and deterministic chaos: Limits of protein folding simulations and calculations. Chaos Solitons Fractals 1(4):375-382

Boltzmann L (1877) Über die Beziehung zwischen dem zweiten Hauptsatze des mechanischen Wärmetheorie und der Wahrscheinlichkeitsrechnung, respective den Sätzen über das Wärmegleichgewicht. Kk Hof-und Staatsdruckerei

Braverman MS, Yampolsky M (2008) Computability of Julia sets. Mosc Math J 8(2):185-231

Bressloff PC, Cowan JD, Golubitsky M, Thomas PJ, Wiener MC (2001) Geometric visual hallucinations, Euclidean symmetry and the functional architecture of striate cortex. Phil Trans R Soc Lond B 356(1407):299-330

Bressloff PC, Cowan JD, Golubitsky M, Thomas PJ, Wiener MC (2002) What geometric visual hallucinations tell us about the visual cortex. Neural Comput 14(3):473-491

Brooks R, Matelski JP (1981) The dynamics of 2-generator subgroups of PSL (2, C). In Riemann surfaces and related topics: Proceedings of the 1978 Stony Brook Conference, Ann of Math Stud 97:65-71

Buterin V (2014) A next-generation smart contract and decentralized application platform. White paper. https://github.com/ethereum/wiki/wiki/White-Paper

Capra F, Luisi PL (2014) The systems view of life: A unifying vision. Cambridge University Press.

Carnot S (1824) Reflections on the motive power of fire, and on machines fitted to develop that power. Bachelier, Paris

Casino F, Dasaklis TK, Patsakis C (2019) A systematic literature review of blockchain-based applications: current status, classification and open issues. Telematics Inform 36:55-81

Chay TR, Fan YS, Lee YS (1995) Bursting, spiking, chaos, fractals, and universality in biological rhythms. Int J Bifurcat Chaos 5(03):595-635

Chen L, Chen L, Jordan S, Liu YK, Moody D, Peralta R, Smith-Tone D (2016) Report on post-quantum cryptography (Vol. 12). US Department of Commerce, National Institute of Standards and Technology

Cheng X, Ferrell JE (2019) Spontaneous emergence of cell-like organization in Xenopus egg extracts. Science 366(6465):631-637

Christidis K, Devetsikiotis M (2016) Blockchains and smart contracts for the internet of things. Ieee Access 4:2292-2303

Chua LO, Huynh LT (1992) Bifurcation analysis of Chua's circuit, Proceedings of the 35th Midwest Symposium on Circuits and Systems, Washington, DC, USA, 1992, pp. 746-751

Clausius R (1850) Über die bewegende Kraft der Wärme und die Gesetze, welche sich daraus für die Wärmelehre selbst ableiten lassen. Ann Phys 155(3):368-397

Clausius R (1865) Über verschiedene für die Anwendung bequeme Formen der Hauptgleichungen der mechanischen Wärmetheorie. Ann Phys 201(7):353-400

Cleland CE, Chyba CF (2002) Defining 'life.' Orig Life Evol Biosph 32(4):387-393

Coleman BD, Swigon D, Tobias I (2000) Elastic stability of DNA configurations. II. Supercoiled plasmids with self-contact. Phys Rev E 61(1):759

Conway J (1970) The game of life. Sci Am 223(4):4

Corea F (2019) The convergence of AI and blockchain. In Applied Artificial Intelligence: Where AI Can Be Used In Business (pp. 19-26). Springer, Cham.

Crevier DW, Meister M (1998) Synchronous period-doubling in flicker vision of salamander and man. J Neurophysiol 79(4):1869-1878

Crosby M, Pattanayak P, Verma S, Kalyanaraman V (2016) Blockchain technology: Beyond bitcoin. Applied Innovation 2(6-10):71

Crutchfield JP (1994) The calculi of emergence: computation, dynamics and induction. Physica D 75(1-3):11-54

Cushing JM (1977) Periodic time-dependent predator-prey systems. SIAM J Appl Math 32(1):82-95

Dannen C (2017) Introducing Ethereum and Solidity, vol 1. Apress, Berkeley

Darwin, C. (1859). On the Origin of Species by Means of Natural Selection Or the Preservation of Favoured Races in the Struggle for Life. John Murray, London, pp 502

Davies PC, Rieper E, Tuszynski JA (2013) Self-organization and entropy reduction in a living cell. Biosystems 111(1):1-10 
De Wolf T, Holvoet T (2004) Emergence versus self-organisation: Different concepts but promising when combined. In International workshop on engineering self-organising applications (pp. 1-15). Springer, Berlin, Heidelberg

Devaney RL (1986) An introduction to chaotic dynamical systems. Benjamin/Cummings, Menlo Park, Calif Dinh TN, Thai MT (2018) AI and blockchain: A disruptive integration. Computer 51(9):48-53

Douady A, Hubbard JH (1984) Etude dynamique des polynômes complexes. Prépublications mathémathiques d'Orsay $2 / 4$

Douady S, Fauve S (1988) Pattern selection in Faraday instability. EPL (Europhysics Letters) 6(3):221

Einstein A (1917) Zum Quantensatz von Sommerfeld und Epstein. Verh Deutsch Phys Ges 19:82-92

Elluswamy AK, Bauch M, Payne C, Karpathy A, Shroff D, Ramanandan A, Hakewill JRH (2020) "Predicting Three-Dimensional Features For Autonomous Driving", U.S. Patent Application No. US 2020/0249685 A1. Washington, DC: U.S. Patent and Trademark Office

England JL (2013) Statistical physics of self-replication. J Chem Phys 139(12):09B623_1

Ermentrout GB, Cowan JD (1979) A mathematical theory of visual hallucination patterns. Biol Cybern 34(3):137-150

Eskew MW, Simoyi RH (2017) Lyapunov exponents and the Belousov-Zhabotinsky oscillator: an interactive computational approach. S Afr J Chem 70:82-88

Fong RC, Scheirer WJ, Cox DD (2018) Using human brain activity to guide machine learning. Sci Rep $8(1): 1-10$

Forgacs G, Newman SA (2005) Biological physics of the developing embryo. Cambridge University Press

Gardner M (1970) Mathematical games. Sci Am 222(6):132-140

Garfinkel A, Spano ML, Ditto WL, Weiss JN (1992) Controlling cardiac chaos. Science 257(5074):1230-1235

Gaudenzi R, Burzurí E, Maegawa S, van der Zant HSJ, Luis F (2018) Quantum Landauer erasure with a molecular nanomagnet. Nat Phys 14(6):565-568

Gentili PL, Horvath V, Vanag VK, Epstein IR (2012) Belousov-Zhabotinsky "Chemical Neuron" as a Binary and Fuzzy Logic Processor. IJUC 8(2):177-192

Gibbs JW (1878) On the equilibrium of heterogeneous substances. Am J Sci 96:441-458

Gleeson A (2017) Visual Analytics for Classification of Ethereum Cryptocurrency, Masters thesis, Data Science Institute, Imperial College London.

Goldstein J (1999) Emergence as a construct: History and issues. Emergence 1(1):49-72

Grace K, Salvatier J, Dafoe A, Zhang B, Evans O (2018) When will AI exceed human performance? Evidence from AI experts. J Artif Intell Res 62:729-754

Györgyi L, Field RJ (1992) A three-variable model of deterministic chaos in the Belousov-Zhabotinsky reaction. Nature 355(6363):808-810

Hales TC (2001) The honeycomb conjecture. Discrete Comput Geom 25(1):1-22

Henke H, Robinson PA, Drysdale PM, Loxley PN (2009) Spatiotemporal dynamics of pattern formation in the primary visual cortex and hallucinations. Biol Cybern 101(1):3-18

Hertling P (2005) Is the Mandelbrot set computable?. Math Log Q 51(1):5-18

Hirayama M, Guivant J, Katupitiya J, Whitty M (2019) Path planning for autonomous bulldozers. Mechatronics 58:20-38

Hoekzema R (2015) Mathematical studies of morphology in early life palaeobiology (Doctoral dissertation, University of Oxford)

Hoekzema RS, Brasier MD, Dunn FS, Liu AG (2017) Quantitative study of developmental biology confirms Dickinsonia as a metazoan. Proc Roy Soc B: Biol Sci 284(1862):20171348

Holley J, Jahan I, Costello BDL, Bull L, Adamatzky A (2011) Logical and arithmetic circuits in BelousovZhabotinsky encapsulated disks. Phys Rev E 84(5):056110

Holmes JD, Paterson JR, García-Bellido DC (2019) The trilobite Redlichia from the lower Cambrian Emu Bay Shale Konservat-Lagerstätte of South Australia: systematics, ontogeny and soft-part anatomy. J Syst Palaeontol 18(4):295-334

Hong J, Lambson B, Dhuey S, Bokor J (2016) Experimental test of Landauer's principle in single-bit operations on nanomagnetic memory bits. Sci Adv 2(3):e1501492

Inoue M, Kashima M (1994) Self-organization and entropy decreasing in neural networks. Progress Theoret Phys 92(5):927-938

Jacquin AE (1992) Image coding based on a fractal theory of iterated contractive image transformations. IEEE Trans Image Process 1(1):18-30

Jakobsson M, Juels A (1999) Proofs of work and bread pudding protocols. In Secure information networks (pp. 258-272). Springer, Boston, MA

Jia B, Gu H, Li L, Zhao X (2012) Dynamics of period-doubling bifurcation to chaos in the spontaneous neural firing patterns. Cogn Neurodyn 6(1):89-106 
Johnston D, Yilmaz SO, Kandah J, Bentenitis N, Hashemi F, Gross R, Wilkinson S, Mason S (2014) The General Theory of Decentralized Applications, DApps, GitHub, June 9, 2014. https://github.com/ DavidJohnstonCEO/DecentralizedApplications

Joyce GF (1994) Foreward. In: Deamer DW, Fleischaker GR (eds) Origins of Life: The Central Concepts. Jones and Bartlett, Boston, p xi

Jun Y, Gavrilov M, Bechhoefer J (2014) High-precision test of Landauer's principle in a feedback trap. Phys Rev Lett 113(19):190601

Kleidon A, Lorenz R (2005) 1 entropy production by earth system processes. In Non-equilibrium Thermodynamics and the Production of Entropy (pp. 1-20). Springer, Berlin, Heidelberg

Klüver H (1928) Mescal, and mechanisms of hallucinations. University of Chicago Press

Kondo S, Miura T (2010) Reaction-diffusion model as a framework for understanding biological pattern formation. Science 329(5999):1616-1620

Korzeniewski B (2001) Cybernetic formulation of the definition of life. J Theor Biol 209(3):275-286

Koschmieder EL (1974) Bénard convection. Adv Chem Phys 26:177

Krittanawong C, Rogers AJ, Aydar M, Choi E, Johnson KW, Wang Z, Narayan SM (2020) Integrating blockchain technology with artificial intelligence for cardiovascular medicine. Nat Rev Cardiol 17(1):1-3

Kudrolli A, Gollub JP (1996) Patterns and spatiotemporal chaos in parametrically forced surface waves: a systematic survey at large aspect ratio. Physica D 97(1-3):133-154

Kusiak A (2018) Smart manufacturing. Int J Prod Res 56(1-2):508-517

Lake BM, Ullman TD, Tenenbaum JB, Gershman SJ (2017) Building machines that learn and think like people. Behav Brain Sci 40

Landauer R (1961) Irreversibility and heat generation in the computing process. IBM J Res Dev 5(3): $183-191$

Langton C (1990) Computation at the edge of chaos: Phase transition and emergent computation (No. LA-UR-90-379; CONF-8905201-5). Los Alamos National Lab., NM (USA)

Langton CG (1986) Studying artificial life with cellular automata. Physica D 22(1-3):120-149

Lee MS, Jago JB, García-Bellido DC, Edgecombe GD, Gehling JG, Paterson JR (2011) Modern optics in exceptionally preserved eyes of Early Cambrian arthropods from Australia. Nature 474(7353):631-634

Li L, Ota K, Dong M (2018) Humanlike driving: empirical decision-making system for autonomous vehicles. IEEE Trans Veh Technol 67(8):6814-6823

Li JG, Zhan K (2018) Intelligent mining technology for an underground metal mine based on unmanned equipment. Engineering 4(3):381-391

Li TY, Yorke JA (2004) Period three implies chaos. In The Theory of Chaotic Attractors (pp. 77-84). Springer, New York, NY

Lineweaver CH (2005) Cosmological and Biological Reproducibility: Limits on the Maximum Entropy Production Principle. In Non-equilibrium thermodynamics and the production of entropy (pp. 67-77). Springer, Berlin, Heidelberg.

Lorenz EN (1963) Deterministic nonperiodic flow. J Atmos Sci 20(2):130-141

Ma T, Wang S (2004) Dynamic bifurcation and stability in the Rayleigh-Bénard convection. Commun Math Sci 2(2):159-183

Makridakis S, Polemitis A, Giaglis G, Louca S (2018) Blockchain: The next breakthrough in the rapid progress of AI. In Artificial Intelligence-Emerging Trends and Applications, IntechOpen

Mandelbrot BB (1980) Fractal aspects of the iteration of $\mathrm{z} \rightarrow \Lambda \mathrm{z}$ (1-z) for complex $\Lambda$ and $\mathrm{z}$. Ann N Y Acad Sci 357(1):249-259

Mandelbrot BB (1983) The fractal geometry of nature (Vol. 173, p. 51). New York: WH freeman.

Mandelbrot BB (2004) Fractals and Chaos. Springer, New York

Marshall, CR (2006) Explaining the Cambrian "explosion" of animals. Annu Rev Earth Planet Sci $34: 355-384$

Matrosov VV, Kazantsev VB (2011) Bifurcation mechanisms of regular and chaotic network signaling in brain astrocytes. Chaos 21(2):023103

Maurer J, Libchaber A (1979) Rayleigh-Bénard experiment in liquid helium; frequency locking and the onset of turbulence. J Phys Lett 40(16):419-423

May RM (1975) Biological populations obeying difference equations: stable points, stable cycles, and chaos. J Theor Biol 51(2):511-524

May RM (1976) Simple mathematical models with very complicated dynamics. Nature 261(5560):459-467

Mejía J, Michaelian K (2018) Origin of Information Encoding in Nucleic Acids through a Dissipation-Replication Relation. arXiv preprint https://arxiv.org/abs/1804.05939 
Merkle RC (1980) Protocols for public key cryptosystems. Proc IEEE Symposium on Security and Privacy 1980:122-134

Michaelian K (2011) Thermodynamic dissipation theory for the origin of life. Earth Syst Dynam 2:37-51

Murray JD (1990) Discussion: Turing's theory of morphogenesis—its influence on modelling biological pattern and form. Bull Math Biol 52(1-2):117-152

Nakamoto S (2008). Bitcoin: A peer-to-peer electronic cash system. White paper. https://bitcoin.org/ bitcoin.pdf

Nassar M, Salah K, ur Rehman MH, Svetinovic D (2020) Blockchain for explainable and trustworthy artificial intelligence. Wiley Interdiscip Rev: Data Min Knowl Discov 10(1):e1340

National Institute of Standards and Technology (2001) Descriptions of SHA-256, SHA-384 and SHA-512. Technical Report, Gaithersburg, MD, United States, p 50

Newell AC, Shipman PD (2005) Plants and fibonacci. J Stat Phys 121(5-6):937-968

Nicolis G (1990) Chemical chaos and self-organization. J Phys Condens Matter 2(S):SA47

Norta A (2015) Creation of smart-contracting collaborations for decentralized autonomous organizations. In International Conference on Business Informatics Research (pp. 3-17). Springer, Cham.

Nowak MA, May RM (1992) Evolutionary games and spatial chaos. Nature 359(6398):826-829

Orel M, Guna J (2018) Decentralising Virtual Reality. Elektrotehniski Vestnik / Electrotechnical Review $85: 272-278$

Poincaré H (1890) Sur le problème des trois corps et les équations de la dynamique. Acta Math 13(1):A3-A270

Prigogine I, Lefever R (1968) Symmetry breaking instabilities in dissipative systems. II The Journal of Chemical Physics 48(4):1695-1700

Prigogine I, Nicolis G (1967) On symmetry-breaking instabilities in dissipative systems. J Chem Phys 46(9):3542-3550

Prigogine I, Nicolis G, Babloyantz A (1972) Thermodynamics of evolution I. Phys Today 25(11):23-28

Pineda FJ (1987) Generalization of back-propagation to recurrent neural networks. Phys Rev Lett 59(19):2229

Puthal D, Malik N, Mohanty SP, Kougianos E, Yang C (2018) The blockchain as a decentralized security framework [future directions]. IEEE Consumer Electronics Magazine 7(2):18-21

Rathore S, Pan Y, Park JH (2019) BlockDeepNet: a Blockchain-based secure deep learning for IoT network. Sustainability 11(14):3974

Raven PH, Johnson GB, Mason KA, Losos J, Singer S (2017) Biology, 11th edn. McGraw Hill Education, New York, NY, USA

Rayleigh L (1916) LIX. On convection currents in a horizontal layer of fluid, when the higher temperature is on the under side. London Edinburgh Dublin Philos Mag J Sci 32(192):529-546

Rendell P (2002) Turing universality of the game of life. In Collision-based computing (pp. 513-539). Springer, London

Rennard JP (2002) Implementation of logical functions in the Game of Life. In Collision-based computing (pp. 491-512). Springer, London

Richetti P, Roux JC, Argoul F, Arneodo A (1987) From quasiperiodicity to chaos in the Belousov-Zhabotinskii reaction. II. Modeling and theory. Journal Chem Phys 86(6):3339-3356

Rössler OE (1976) Chemical turbulence: chaos in a simple reaction-diffusion system. Zeitschrift für Naturforschung A 31(10):1168-1172

Rössler OE, Wegmann K (1978) Chaos in the Zhabotinskii reaction. Nature 271(5640):89-90

Sagan C (1970) Life, in: The Encyclopaedia Britannica, William Benton, London.

Salah K, Rehman MHU, Nizamuddin N, Al-Fuqaha A (2019) Blockchain for AI: Review and open research challenges. IEEE Access 7:10127-10149

Sayeed S, Marco-Gisbert H (2019) Assessing blockchain consensus and security mechanisms against the $51 \%$ attack. Appl Sci 9(9):1788

Scherer,M (2017). Performance and Scalability of Blockchain Networks and Smart Contracts. Dissertation, Umeå University, Umeå, Sweden. Retrieved from http://urn.kb.se/resolve?urn=urn:nbn:se:umu: diva-136470

Schiff SJ, Jerger K, Duong DH, Chang T, Spano ML, Ditto WL (1994) Controlling chaos in the brain. Nature 370(6491):615-620

Schollmeier R (2001) A definition of peer-to-peer networking for the classification of peer-to-peer architectures and applications. In Proceedings First International Conference on Peer-to-Peer Computing, pp. 101-102, IEEE

Schrödinger E (1944) What Is Life? The physical aspect of the living cell and mind. Cambridge University Press, Cambridge 
Sepkoski Jr JJ (1979) A kinetic model of Phanerozoic taxonomic diversity II. Early Phanerozoic families and multiple equilibria. Paleobiology 222-251

Shannon CE (1948) A mathematical theory of communication. Bell Syst Tech J 27(3):379-423

Shaw R (1984) The Dripping Faucet as a Model Chaotic System. Aerial, Santa Cruz, p 111

Shen B, Dong L, Xiao S, Kowalewski M (2008) The Avalon explosion: evolution of Ediacara morphospace. Science 319(5859):81-84

Singh SK, Rathore S, Park JH (2020) BlockIoTIntelligence: A blockchain-enabled intelligent IoT architecture with artificial intelligence. Futur Gener Comput Syst 110:721-743

Solé RV, Miramontes O (1995) Information at the edge of chaos in fluid neural networks. Physica D 80(1-2):171-180

Solomon EP, Martin CE, Martin DW, Berg LR (2018) Biology, 11th edn. Cengage, Boston, MA, USA

Su Y, Liu Y, Zhou Y, Yuan J, Cao H, Shi J (2019) Broadband LEO satellite communications: Architectures and key technologies. IEEE Wirel Commun 26(2):55-61

Swenson R (1989) Emergent attractors and the law of maximum entropy production: foundations to a theory of general evolution. Syst Res 6(3):187-197

Szabo N (1997) Formalizing and securing relationships on public networks. First Monday 2(9). https://doi. org/10.5210/fm.v2i9.548

Szilard L (1929) Über die Entropieverminderung in einem thermodynamischen System bei Eingriffen intelligenter Wesen. Z Phys 53(11-12):840-856

Talpes E, Sarma DD, Venkataramanan G, Bannon P, McGee B, Floering B, Sachdev GS (2020) Compute Solution for Tesla's Full Self-Driving Computer. IEEE Micro 40(2):25-35

Tereshko V (2011) Control and Identification of Chaotic Systems by Altering the Oscillation Energy. Chaotic Syst 135

Tompkins N, Li N, Girabawe C, Heymann M, Ermentrout GB, Epstein IR, Fraden S (2014) Testing Turing's theory of morphogenesis in chemical cells. Proc Nat Acad Sci 111(12):4397-4402

Torbensen K, Rossi F, Pantani OL, Ristori S, Abou-Hassan A (2015) Interaction of the Belousov-Zhabotinsky reaction with phospholipid engineered membranes. J Phys Chem B 119(32):10224-10230

Torbensen K, Rossi F, Ristori S, Abou-Hassan A (2017) Chemical communication and dynamics of droplet emulsions in networks of Belousov-Zhabotinsky micro-oscillators produced by microfluidics. Lab on a Chip 17(7):1179-1189

Turcotte DL (1997) Fractals and chaos in geology and geophysics. Cambridge University Press

Turing AM (1936) On computable numbers, with an application to the Entscheidungsproblem. J Math 58(345-363):5

Turing AM (1952) The chemical basis of morphogenesis. Phil Trans R Soc Lond B 237:37-72

Tyson JJ, Alexander KA, Manoranjan VS, Murray JD (1989) Spiral waves of cyclic AMP in a model of slime mold aggregation. Physica D 34(1-2):193-207

Valenta M, Sandner P (2017) Comparison of ethereum, hyperledger fabric and corda. [ebook] Frankfurt School, Blockchain Center.

Valentine JW (2002) Prelude to the Cambrian explosion. Annu Rev Earth Planet Sci 30(1):285-306

Van der Maas HL, Verschure PF, Molenaar PC (1990) A note on chaotic behavior in simple neural networks. Neural Netw 3(1):119-122

Venturi D, Wan X, Karniadakis GE (2010) Stochastic bifurcation analysis of Rayleigh-Bénard convection. J Fluid Mech 650:391

von Helmholtz H (1882) Die thermodynamik chemischer Vorgänge. Sitzungsberichten der Akademie der Wissenschaften zu Berlin 1:23

Von Neumann J, Burks AW (1966) Theory of Self-Reproducing Automata. Univ Illinois Press

Wagner C, Müller HW, Knorr K (1999) Faraday waves on a viscoelastic liquid. Phys Rev Lett 83(2):308

Wang J, Ma Y, Zhang L, Gao RX, Wu D (2018) Deep learning for smart manufacturing: Methods and applications. J Manuf Syst 48:144-156

Wang LP, Pichler EE, Ross J (1990) Oscillations and chaos in neural networks: an exactly solvable model. Proc Natl Acad Sci 87(23):9467-9471

Wang X (1991) Period-doublings to chaos in a simple neural network: An analytical proof. Complex Syst 5(4):425-444

Wiener N (1948) Cybernetics or control and communication in the animal and the machine. Technology Press

Wolfram S (1984) Cellular automata as models of complexity. Nature 311(5985):419-424

Xiao S, Laflamme M (2009) On the eve of animal radiation: phylogeny, ecology and evolution of the Ediacara biota. Trends Ecol Evol 24(1):31-40

Yaga D, Mell P, Roby N, Scarfone K (2019). Blockchain technology overview. arXiv preprint https://arxiv. org/abs/1906.11078 
Yamagishi MEB, Shimabukuro AI (2008) Nucleotide frequencies in human genome and Fibonacci numbers. Bull Math Biol 70(3):643-653

Zenil H (2015) Algorithmicity and programmability in natural computing with the Game of Life as in silico case study. J Exp Theor Art Intell 27(1):109-121

Zhabotinsky AM (1964) Periodical oxidation of malonic acid in solution (a study of the Belousov reaction kinetics). Biofizika 9:306-311

Zhang G, Li T, Li Y, Hui P, Jin D (2018) Blockchain-based data sharing system for AI-powered network operations. J Commu Info Net 3(3):1-8

Zhuravlev AY, Riding R, eds (2001) The Ecology of the Cambrian Radiation. New York: Columbia Univ. Press. pp 525

Publisher's Note Springer Nature remains neutral with regard to jurisdictional claims in published maps and institutional affiliations. 